\title{
Zebrafish yolk syncytial nuclei migrate along a dynamic microtubule network
}

\author{
Zhonghui Fei $^{1^{*}}$, Koeun Bae ${ }^{1 *}$, Serge E. Parent ${ }^{1}$, Katharine Goodwin ${ }^{2,3}$, Guy Tanentzapf ${ }^{2}$ \\ and Ashley E.E. Bruce ${ }^{1+}$
}

1 Department of Cell and Systems Biology

University of Toronto

Toronto, ON M5S 3G5

2 Life Sciences Institute

Vancouver Campus

2350 Health Sciences Mall

Vancouver, BC Canada V6T 1 Z3

3 Current address:

Department of Chemical \& Biological Engineering

Princeton University

303 Hoyt Laboratory

William Street

Princeton, NJ 08544

* These authors contributed equally to this work.

†Corresponding author

ashley.bruce@utoronto.ca

orcid.org/0000-0002-0567-2928

Running title: Yolk syncytial nuclear migration

Keywords: zebrafish, epiboly, yolk syncytial layer, yolk syncytial nuclei, microtubule, LINC complex 
Summary Statement

2 Analysis of yolk syncytial nuclear migration during zebrafish epiboly reveals that nuclei migrate

3 along and largely beneath a dynamically yolk microtubule network.

6 Abstract

7 In teleosts, the yolk syncytial layer is a multinucleate syncytium that functions as an

8 extraembryonic signaling center to pattern the mesendoderm, coordinate morphogenesis and

9 supply nutrients to the embryo. The zebrafish is an excellent system for studying this

10 morphogenetically active tissue. The external yolk syncytial nuclei (e-YSN) undergo microtubule

11 dependent epiboly movements that distribute the nuclei over the yolk. How e-YSN epiboly

12 proceeds, and what role the yolk microtubule network plays is not understood but currently it is

13 proposed that e-YSN are pulled vegetally as the microtubule network shortens from the vegetal

14 pole. Data from our live imaging studies suggest that the yolk microtubule network is dismantled

15 from the animal and vegetal regions and show that a region of stabilized microtubules forms

16 before nuclear migration begins. e-YSN do not appear to be pulled vegetally but rather move

17 along a dynamic microtubule network. We also show that overexpression of the KASH domain

18 of Syne2a impairs e-YSN movement, implicating the LINC complex in e-YSN migration. This

19 work provides new insights into the role of microtubules in morphogenesis of an extraembryonic

20 tissue. 


\section{Introduction}

27 Embryonic development involves coordinated cell shape changes and movements to establish the

28 adult body plan and developmental programs are inextricably linked to embryo architecture. In

29 teleosts, the yolk syncytial layer (YSL) is a conserved and essential extraembryonic signaling

30 center which contains transcriptionally active yolk syncytial nuclei (YSN). The YSL has

31 numerous functions including induction and patterning of mesendoderm, coordination of epiboly

32 movements and provision of nutrients to the embryo (Mizuno et al. 1999; Feldman et al. 1998;

33 Ober \& Schulte-Merker 1999; Rodaway et al. 1999; Gritsman et al. 2000; Koos \& Ho 1998;

34 Thomas 1968; Ho et al. 1999; Sirotkin et al. 2000; Fekany et al. 1999; Fekany-Lee et al. 2000;

35 Chen \& Kimelman 2000). In addition, the YSL undergoes surprisingly dynamic shape changes

36 during development, making it an excellent system to gain new insights into morphogenesis of an

37 extraembryonic tissue (Carvalho et al. 2009; D'Amico \& Cooper 2001; Virta \& Cooper 2011).

38 YSL functions rely upon YSN transcription (Chen \& Kimelman 2000; Xu et al. 2012) and a

39 population of YSN undergo active epiboly movements which distributes the nuclei over the yolk

40 surface (D'Amico \& Cooper 2001; Carvalho et al. 2009). When the nuclei are not properly

41 distributed, epiboly and patterning are adversely affected (Carvalho \& Heisenberg 2010; Xu et al.

42 2012; Takesono et al. 2012).

44 The YSL forms as a result of meroblastic cleavages which generates the blastoderm on top of a

45 large yolk cell (Carvalho \& Heisenberg 2010; Kimmel \& Law 1985; Trinkaus 1993). In

46 zebrafish, incomplete early cleavages result in marginal blastomeres remaining open to the yolk

47 cell and, around the time of the maternal-zygotic transition, marginal blastomeres release their

48 cytoplasm and nuclei into the previously anuclear yolk cell to form the YSL. The YSL consists of

49 the external-YSL (e-YSL) at the yolk-blastoderm exterior interface while the internal YSL (i-

50 YSL) lies directly beneath the blastoderm (Kimmel \& Law 1985). Yolk syncytial nuclei (YSN)

51 are located in both regions and are referred to as e-YSN and i-YSN (Kimmel et al. 1995) (Fig.

52 1A). YSN undergo several mitotic divisions before they exit the cell cycle (Kane et al. 1992).

53 YSN become enlarged and in some species, have been shown to become polyploid (Bachop and

54 Schwartz, 1974).

56 Epiboly is a major cell movement during teleost development. Epiboly involves the thinning and

57 spreading of a multilayer of cells and the active motive force provided by the YSL is absolutely 
required for this process (Trinkaus 1963). During epiboly, the blastoderm and YSL spread down towards the vegetal pole to cover and enclose the yolk cell by $10 \mathrm{hpf}$ (Fig. 1A). The blastoderm generates all embryonic tissues and it consists of an outer epithelial layer, the enveloping layer (EVL), which is tightly attached at its margin to the yolk cell and covers the underlying deep cells. In zebrafish, epiboly begins at 4.3 hours post-fertilization (hpf), following the cessation of YSN mitotic divisions and lineage specification of the EVL (Kimmel et al. 1995). The e-YSL has been shown to provide mechanical force necessary to drive epiboly via actomyosin motors (Behrndt et al. 2012; Cheng et al. 2004). There is also a distinct longitudinal microtubule array that is often disrupted in embryos with abnormal epiboly (reviewed in Lepage \& Bruce 2010; Bruce 2016). The microtubule network is nucleated from the most vegetally positioned e-YSN in the YSL and oriented along the animal-vegetal axis with the microtubule plus ends extending into the yolk cytoplasmic layer (YCL), which is a thin layer of cytoplasm that surrounds the dense core of yolk granules (Strähle \& Jesuthasan 1993; Solnica-Krezel \& Driever 1994). During epiboly, the microtubule network shortens as the YCL is gradually replaced by the YSL (SolnicaKrezel \& Driever 1994).

The dramatic morphogenetic changes in the YSL and its role in promoting epiboly have been recognized for some time, but much remains to be learned about the mechanisms that drive yolk morphogenesis. The yolk cell microtubules were first implicated in YSL epiboly in studies from Jesuthasan and Strahle (1993) and Solnica-Krezel and Driever (1994). Ultra-violet irradiation of cleavage stage embryos or treatment with the microtubule depolymerizing drug nocodazole resulted in delayed epiboly (Strähle \& Jesuthasan 1993). Solnica-Krezel and Driever (1994) showed that treatment of late blastula stage embryos with nocodazole prevented the e-YSN from moving vegetally during epiboly. In contrast, vegetal movement of the blastoderm was not as strongly impaired, although epiboly movements were slowed. Taxol treatment, which stabilizes microtubules, slowed epiboly of the YSL and blastoderm to similar extents (Solnica-Krezel \& Driever 1994).

Jesuthasan and Strahle (1993) proposed that the yolk microtubule network comprises part of the epiboly motor. They suggested that yolk microtubules act to expand the YSL via microtubule motors moving vegetally along the microtubules, and pulling the attached blastoderm along with 
blastoderm, was completely dependent upon the yolk microtubules. They postulated that the

91 primary function of the network is to move the e-YSN vegetally during epiboly and that epiboly

92 of the blastoderm is independent from epiboly of the e-YSN. They put forward several potential

93 models in which different microtubule motors could provide pulling or pushing forces to move

94 the e-YSN. One model was that, as the yolk microtubules shorten from the vegetally located plus

95 ends, the e-YSN are pulled downwards (Solnica-Krezel \& Driever 1994).

97 The positioning and movement of nuclei is important in a number of developmental contexts

98 (Bone \& Starr 2016). The linker of nucleoskeleton and cytoskeleton (LINC) complex has

99 emerged as an important and conserved component of the nucleus that functions to connect

100 structural elements in the nucleus to the cytoskeleton (Starr \& Fridolfsson 2010). The complex

101 consists of Sad1p/UNC-84 (SUN) and Klarsicht/ANC-1/Syne (KASH) proteins, located in the

102 inner and outer nuclear membranes respectively. The LINC complex is capable of interacting

103 with microtubules, centrosomes, F-actin, intermediate filaments and the microtubule motor

104 proteins dynein and kinesin (Starr \& Fridolfsson 2010; Chang et al. 2015). Well established

105 examples of microtubule based nuclear movement include pronuclear fusion, muscle fiber

106 development, and neuronal interkinetic nuclear migration (Bone \& Starr 2016).

108 How e-YSN move towards the vegetal pole remains unclear. In addition, the dynamics of the 109 yolk microtubule network have not been reported in detail during epiboly. Here we revisit these

110 questions using live imaging and quantitative analyses. We show that the organization of the yolk

111 cell microtubule network undergoes striking changes just prior to e-YSN movement, which have

112 not previously been reported. We observed that e-YSN move vegetally through and largely

113 beneath the microtubule network, in contrast to the current view. In addition, we show that the

114 LINC complex appears to be involved in e-YSN epiboly. We present a new model for e-YSN

115 movement that takes into account the observed changes in microtubule dynamics and proposes

116 that microtubule motor proteins interact directly with e-YSN via the LINC complex to drive

117 vegetal e-YSN movement.

118

119 Results

120 The yolk microtubule network undergoes dynamic changes during epiboly

121 The yolk microtubule network covers approximately 400 microns along the animal-vegetal (A-V) 
122 axis of the exposed multinucleate yolk cell at the start of epiboly (Kimmel et al. 1995). The yolk

123 cell size is about ten times that of a typical cell, which may enable the formation of microtubule

124 patterns that are not possible in smaller cells due to the differences in scale. To learn more about

125 how e-YSN use microtubules for their movement, we examined the organization and dynamics of

126 the network in live embryos. To accomplish this we used embryos from the previously

127 characterized transgenic line Tg:(XlEefla1:dclk2DeltaK-GFP) in which microtubules are

128 indirectly labeled via binding of a microtubule associated protein fused to GFP (Sepich et al.

129 2011). For our analyses, we divided epiboly into early (dome to shield, 4.3-6 hpf), mid- (shield-

$13075 \%$ epiboly, 6-8 hpf) and late (75\% epiboly-bud, 8-10 hpf) stages (Fig. 1A).

132 At sphere stage, prior to epiboly initiation, the yolk cell microtubule network in both transgenic

133 and alpha-tubulin stained embryos was nucleated from microtubule organizing centers associated

134 with a subset of the most vegetally positioned e-YSN (Fig. 1B). Based on appearance, we refer to

135 microtubules nucleated from an individual e-YSN as a 'branch' since they broadened as they

136 extended vegetally (Fig. 1B). We also observed gaps between microtubule branches emanating

137 from adjacent e-YSN (Fig. 1D'). Although the yolk microtubule network originated from the e-

138 YSN, the widening of the branches vegetally suggested that vegetal microtubules were unlikely

139 to be nucleated entirely from e-YSN associated microtubule organizing centers, given the size of

140 the yolk cell and based on other analyses described below. This observation is consistent with

141 work in Xenopus eggs showing that microtubules can be nucleated from existing microtubules

142 and that interphase cytoplasm has the ability to support spontaneous microtubule growth

143 (Ishihara et al. 2014).

145 To examine microtubule network dynamics, we generated low magnification confocal time-lapse

146 movies of live transgenic embryos. In movies that captured the last YSN division during sphere

147 stage, we observed that the division was accompanied by what resembled a wave of microtubule

148 bundling followed by the re-establishment of the network from the e-YSN (Movie 1). This

149 observation supports the idea that the e-YSN provide the polarity and the basic scaffold upon

150 which the yolk microtubule network is built. We also note that this type of microtubule network

151 is not observed in regularly sized cells, which would be encompassed within the width of a single

152 microtubule branch. 
154 The blastoderm began to spread vegetally shortly after the final YSN division. During early 155 epiboly, a region of reduced fluorescence became increasingly apparent between the blastoderm 156 margin and the vegetal microtubules in the YCL (Fig. 1C', bracket). During mid-epiboly, this

157 band of reduced fluorescence, which we refer to as the dim zone (DZ), moved vegetally ahead of 158 the blastoderm. On close observation, it was apparent that microtubules were present in the DZ

159 but they appeared to be more diffuse and microtubule fragments were rarely observed, suggesting 160 a change in microtubule organization (Fig. 1E', inset). More intensely fluorescent, and 161 potentially bundled, microtubules were apparent vegetal to the DZ. We also observed that in 162 some dclk2DeltaK-GFP expressing embryos, microtubules cleared from the vegetal area of the 163 yolk cell (Fig. 1E', arrowhead). We postulated that this could result from depolymerization at the 164 vegetal pole or from upward movement of the network towards the animal pole, or from a 165 combination of the two. Around 60\% epiboly, individual e-YSN began to move vegetally (Fig.

$\left.1661 \mathrm{C}^{\prime \prime}\right)$ which will be described below. As epiboly progressed the gaps between microtubule 167 branches were less apparent (for example Fig. 1D ','). During late epiboly, the DZ became less 168 distinct as the network became disorganized (Fig. 1C',', D',', E',', ). Our observations were 169 consistent with reports that the microtubules shorten over the course of epiboly (Solnica-Krezel

$170 \&$ Driever 1994) but the DZ in the upper region of the yolk suggested that depolymerization from 171 the vegetal pole might not be the exclusive mechanism. The dim zone moves vegetally during epiboly

174 To confirm and quantify our observations, fluorescence intensity measurements and kymographs 175 of dclk2DeltaK-GFP time-lapse movies were generated (Fig. 2A,B). We were able to define the $176 \mathrm{DZ}$ as a minimum between the blastoderm and the vegetal mass of microtubules in the yolk cell 177 in A-V fluorescence profile plots taken from the center of the embryo (Fig. 2A). The fluorescence 178 profiles revealed that the signal was high and relatively noisy in the blastoderm, as well as in the 179 vegetal region of the yolk cell. By contrast, the DZ was characterized as a valley between the 180 blastoderm and vegetal pole in which the fluorescence profile was smooth. These observations 181 are consistent with the more diffuse organization of microtubules and an overall reduction in 182 microtubules in the DZ. By tracking the DZ over time we observed that in all cases the DZ 183 moved towards the vegetal pole (Fig. 2B). The mean speed of the DZ was constant at 184 approximately $0.826 \mu \mathrm{m} / \mathrm{min} \pm 0.146 \mu \mathrm{m} / \mathrm{min}$, until late epiboly when the DZ could no longer be 185 reliably detected. Mean speeds for each embryo are given in Table 1. The speed of the DZ is 
186

187

188

189

190

191

192

193

roughly similar to the reported rate of $15 \%$ per hour for blastoderm epiboly from shield to bud stage (Kimmel et al. 1995), which is approximately equivalent to $0.75 \mu \mathrm{m} / \mathrm{min}$.

Particle Image Velocimetry (PIV) analysis of four dclk2DeltaK-GFP time-lapse movies was done to further analyze microtubule movements (Fig. 2C,D). This analysis focused on the vegetal microtubules just below the DZ from early epiboly through the start of mid-epiboly. As expected, movement predominated along the A-V axis, rather than laterally. In embryos 1-3, there was greater mean displacement upwards towards the animal pole than downwards towards the vegetal pole (Fig. 2C red boxes, D). Embryo 4 differed in that there was greater mean displacement towards the vegetal pole than towards the animal pole throughout the time lapse (Fig. $2 \mathrm{C}, \mathrm{D})$. In all 4 embryos, the mean A-V speeds were an order of magnitude slower than the speed of the DZ. These findings suggest that the predominant event from early to mid-epiboly is the change in microtubule dynamics in the DZ and its vegetal progression. Microtubules within the DZ appeared to become more diffuse and the fluorescence was reduced. These observations suggest that the microtubule network is being dismantled, at least in part, from the top as the DZ moves vegetally. Below the DZ, fluorescence intensity was higher than within the $\mathrm{DZ}$ and microtubules were clearly visible. We hypothesized that this subset of microtubules might be more stable due to the accumulation of post-translational modifications.

\section{Detyrosinated tubulin is present in a subset of microtubules during mid-epiboly}

To investigate the potential heterogeneity of the yolk cell network, we performed whole-mount antibody staining for detyrosinated tubulin, which is associated with longer-lived microtubules in vivo (Song \& Brady 2014; Webster et al. 1987; Kreis 1987). Detyrosinated microtubules are typically present in cells, though often at very low levels, leading to the convention that detyrosinated microtubules are defined by detection over background using anti-detyrosinated tubulin antibodies (Bulinski \& Gundersen 1991). Antibody staining in the yolk cell was technically challenging due to fixation, penetration and yolk trapping issues. Detyrosinated tubulin was detected by antibody staining in a subset of microtubules in the central region of the yolk of mid-epiboly stage embryos but was undetectable in sphere stage embryos (Fig. 3).

Detyrosinated microtubules were located vegetally to the DZ and they did not extend to the vegetal pole. At $60 \%$ epiboly, migrating e-YSN could be seen about to enter this region (Fig. 3B, arrowhead). The antibody staining results were consistent with the idea that a subpopulation of 
stabilized detyrosinated microtubules is present during mid-epiboly outside the DZ.

\section{EB3-GFP reveals widespread microtubule polymerization during early epiboly}

221 To further characterize microtubule dynamics during epiboly, we investigated polymerization of

222 the yolk cell microtubule network in live embryos by injecting eb3-gfp RNA into 1-cell stage

223 embryos. EB3 is a microtubule plus end tracking protein that binds to actively growing

224 microtubule plus ends and thus can provide information about the location and rate of

225 microtubule growth as well as the polarity of the network (Stepanova et al. 2003). Embryos

226 injected with eb3-gfp RNA were examined by confocal time-lapse microscopy from sphere to

$22785 \%$ epiboly. EB3-GFP fluorescent streaks (or comets) indicate active microtubule

228 polymerization from the plus end.

230 Surprisingly, large numbers of EB3-GFP comets were visible throughout the yolk cell at sphere

231 stage, indicating extensive microtubule growth (Fig. 4A, Movie 2). Some EB3-GFP comets

232 clearly initiated at centrosomes associated with e-YSN while others could not be traced back to

233 the e-YSN, providing support for the presence of non-centrosomal microtubules. EB3-GFP

234 comets spread downwards towards the vegetal pole, consistent with the network having uniform

235 polarity with microtubule plus ends extending vegetally (Solnica-Krezel \& Driever 1994). Some

236 comets curved laterally, consistent with morphology of the feather-like branches observed in

237 dclk2DeltaK-GFP embryos. EB3-GFP comets were observed throughout early epiboly stages.

238 Strikingly, during mid-epiboly the comets began to diminish and were largely undetectable in the

239 YCL at late epiboly stages (Fig. 4A). Some EB3-GFP comets were still observed in the e-YSL,

240 positioned close to the blastoderm, animal to the DZ. PIV analysis of a single plane time-lapse

241 movie focused on the upper region of the yolk cell was consistent with our qualitative

242 observations, showing that the predominant movement of EB3-GFP comets was along the A-V

243 axis and directed towards the vegetal pole, with an average speed of $3.6 \mu \mathrm{m} / \mathrm{min}$ (Fig. 4B). The

244 small amount of lateral movement might be explained by the feather-like shape of the

245 microtubule branches.

247 To relate microtubule polymerization dynamics to nuclear movement, e-YSN in EB3-GFP

248 movies were examined. Visible as non-fluorescent ovals, e-YSN could be seen to move out from

249 regions where EB3-GFP puncta were being produced (Movie 3). As e-YSN moved vegetally 
EB3-GFP comets were visible behind them. Multiple e-YSN could be observed to move along microtubule branches being nucleated from stationary microtubule organizing centers. Currently it is not clear what causes the reduction in EB3-GFP comets and their confinement to the e-YSL. The reduction in puncta occurred during mid-epiboly, around the time that the DZ formed and detyrosinated microtubules were first detected. Intriguingly, these two events take place around that time that e-YSN begin to migrate. The temporal correlation between these events suggests that they might be linked and important for e-YSN movement.

\section{e-YSN move along and beneath the yolk microtubule network}

259 The e-YSN start to move vegetally during mid-epiboly, after the formation of the shield (the zebrafish organizer) but what triggers the movement is not known (Solnica-Krezel \& Driever 1994). We identified changes in the yolk microtubule network during mid-epiboly, before the eYSN start to migrate. We postulated that initiation of e-YSN movement could be related to these changes. Thus, we sought to understand the relationship between the e-YSN and the yolk microtubules in more detail.

In low power time lapse movies of Tg:(dclk2DeltaK-GFP) and Tg:(X1Eefla1:GFP-tuba81) embryos, migrating e-YSN were visible as non-fluorescent ovals surrounded by fluorescent microtubules (Figs. 1C', 5A). Interestingly, as e-YSN began to migrate, they were often seen to move along the same trajectory. In a representative example, a single e-YSN moved vegetally and then shifted slightly medially, at which point a second e-YSN fell in line behind it and then a third e-YSN joined the line as if on a track (Fig. 5A). e-YSN often appeared to be linked, similar to previous reports of e-YSN chains connected by nuclear bridges (D'Amico \& Cooper 2001). The strings of e-YSN were associated with microtubule branches extending from the YSL, confirming our observations that e-YSN move along EB3-GFP branches. Migrating e-YSN moved through the DZ, where the branches were less distinct, and then into the dense vegetal network of microtubules in the lower yolk. moved on average at approximately $1.936 \pm 0.025 \mu \mathrm{m} / \mathrm{min}$ (see Table 2 for mean speeds per embryo; Table S1 mean speeds per nuclei). Nuclei did not appear to move at a uniform speed 
282 speed. These bursts of speed did not occur simultaneously, consistent with each e-YSN moving

283 independently. Interestingly, the average speed was faster than blastoderm and DZ epiboly

284 supporting the proposal that e-YSN epiboly is independent from blastoderm epiboly (Solnica-

285 Krezel \& Driever 1994).

287 e-YSN movement was further examined using spinning disk confocal time-lapse microscopy of

288 Tuba81-GFP expressing embryos in which nuclei were fluorescently labeled with H2A-GFP.

289 Consistent with Solnica-Krezel and Driever (1994) and our low power time-lapse movies, as the

290 e-YSN began to move they typically underwent a shape change from round to elongate with the

291 pointed end indicating the direction of movement (Fig. 5C). This shape change was most

292 prominent around 60\% epiboly, when the movement initiated. As the nuclei moved they

293 exhibited small bulges and contractions on their surface (Movie 4). We confirmed that nuclear

294 movements were continuous with short bursts of faster movement and migrating e-YSN were not

295 observed to move backwards. Nuclei moved within individual microtubule branches and were

296 not seen to cross the gap between branches. To understand the 3-dimensional relationship

297 between the yolk microtubules and the e-YSN, we inspected Z-stacks from spinning disk

298 confocal movies, and observed that the bulk of the microtubule network was more superficially

299 located than the e-YSN (Fig. 5D). In the deepest e-YSN focal plane, microtubules were visible

300 around the nuclei but were otherwise sparse compared to more superficial planes.

301

302

\section{Over-expression of a dominant-negative KASH construct disrupts e-YSN movement}

303 There are several known mechanism whereby microtubules mediate nuclear migration

304 (Gundersen \& Worman 2013). In large cells, microtubules can exert pulling forces on

305 centrosomes, which often involves cortically anchored dynein. Another method, common during

306 developmental processes and exemplified by female pronuclear migration, involves nuclear

307 envelope associated motor proteins 'walking' the nucleus down the microtubules (Gundersen \&

308 Worman 2013). Given our observation that the e-YSN move past and beneath the yolk

309 microtubule network, we hypothesized that motor proteins move the e-YSN by directly

310 associating with them. In addition, the formation of the DZ and the observation that e-YSN move

311 through this region appears incompatible with vegetally anchored motor proteins pulling the e-

312 YSN down the length of the yolk cell. We explored the possibility that the LINC complex, which

313 is known to interact with microtubules and microtubule motor proteins (Starr \& Fridolfsson 
314 2010), was involved in e-YSN migration. Work in other systems, including the zebrafish retina,

315 showed that overexpression of the KASH domain alone can impair nuclear movement by acting

316 in a dominant-negative fashion to disrupt interactions between the LINC complex and

317 cytoskeletal components or motor proteins (Tsujikawa et al. 2007; Grady et al. 2005).

319 To test the potential role of the LINC complex in e-YSN nuclear movement, we overexpressed

320 the KASH domain of zebrafish Syne2a (C-syne2a) (Tsujikawa et al. 2007). Embryos were

321 injected at the 1-cell stage with a mixture of $c$-syne $2 a$ and $h 2 a-g f p$ RNA or with $h 2 a-g f p$ RNA

322 alone as a control. Confocal time-lapse microscopy was performed on injected embryos during

323 mid-epiboly stages. In control embryos, e-YSN elongated in the direction of movement (Fig. 6

324 cell \#1, Movie 5) as they moved towards the vegetal pole, as described above. In c-syne2a

325 injected embryos, epiboly was overtly normal, however there were defects in the appearance and

326 behavior of the e-YSN. The e-YSN did not become elongated to point in the direction of

327 movement but were more globular in shape. Furthermore, instead of moving vegetally some e-

328 YSN in c-syne $2 a$ injected embryos rotated sideways such that their movement was perpendicular

329 to the A-V axis (Fig. 6 cells \#2 and \#3, Movie 6). Other e-YSN moved animally and some were

330 overrun by the advancing blastoderm margin (Fig. 6 cell \#1). These behaviors were not observed

331 in control embryos. In c-syne $2 a$ injected embryos, most e-YSN were still carried vegetally,

332 though in a less directed manner and we hypothesize that this movement is passive as a result of

333 expansion of the YSL. These results suggest that SUN-KASH proteins are involved in directed e-

334 YSN movement.

336 We postulate that the LINC complex interacts directly with microtubule motors to move the e-

337 YSN. Typically, centrosomes remain associated with nuclei as they migrate (Dupin \& Etienne-

338 Manneville 2011). Centrosomes can be located in front of the nucleus as it moves, with force

339 transmitted to the nucleus via microtubules anchored at the centrosome (Solecki et al. 2004). In

340 this scenario, dynein, a minus end directed motor, would be expected to drive nuclear movement

341 in the yolk. However, the centrosome does not always lead the migration (Umeshima et al. 2007)

342 and if this were the case, given the polarity of the yolk microtubule network, the plus end directed

343 motor kinesin would be expected to be involved. To determine the location of the centrosome

344 during e-YSN migration, we performed gamma-tubulin antibody staining. Gamma-tubulin was

345 detected in association with e-YSN in the YSL at sphere and dome stages (not shown) but we 
346 were unable to detect gamma-tubulin in the yolk at later stages, due to background fluorescence.

347 Our attempts to visualize the centrosome in live embryos during late epiboly by injecting RNA

348 encoding Centrin-GFP or Gamma-tubulin-GFP were also unsuccessful. Thus, additional data are

349 required to determine the position of the centrosome during nuclear migration as well as the

350 motor protein involved.

351

\section{Discussion}

353 The YSL is critically important for patterning and morphogenesis of the blastoderm. Previous

354 work demonstrated that e-YSN undergo active microtubule dependent epiboly movements during

355 gastrulation (Solnica-Krezel \& Driever 1994; Carvalho et al. 2009; D'Amico \& Cooper 2001).

356 How epiboly of the e-YSN proceeds, and what role the yolk microtubule network performs

357 during the process is not understood. Here we find that the e-YSN move along and largely

358 beneath the cortical microtubule network. In addition, we identified changes in the structure and

359 dynamics of the network that occur prior to e-YSN movement. Our results also implicate the

360 LINC complex in e-YSN migration, confirming that microtubules are functionally involved.

Yolk Microtubule Organization and Dynamics

363 The e-YSN nucleate initially non-overlapping microtubule branches which broaden and extend to

364 the vegetal pole of the yolk cell. We propose that parts of the equatorial and vegetal network

365 contain non-centrosomal microtubules. Non-centrosomal microtubules can be nucleated from

366 golgi membranes, the nuclear envelop or from existing microtubules (Petry \& Vale 2015; Lüders

$367 \&$ Stearns 2007). We think it likely that vegetal microtubules are nucleated from existing

368 microtubules, as reported for interphase Xenopus egg extracts (Ishihara et al. 2014). Although the

369 zebrafish embryo is not as large as the Xenopus egg $(750 \mu \mathrm{m}$ versus $1250 \mu \mathrm{m})$, in both cases a

370 yolk microtubule network forms over a distance that is much greater than a single cell. In

371 addition, the smaller zebrafish embryo evolved from a large-egged ancestor similar to present day

372 frogs (Cooper \& Virta 2007), suggesting that mechanisms for generating the yolk microtubule

373 network may be conserved. The Xenopus work also showed that microtubule based nucleation

374 produces a network of parallel microtubules with uniform polarity (Petry et al. 2013), as is the

375 case in the zebrafish yolk cell. Proof of this hypothesis will require the detection of gamma-

376 tubulin at branched nucleation sites on yolk microtubules. 
378 The yolk microtubule network has been assumed to be established prior to the start of epiboly and to be progressively shortened from the vegetal pole (Solnica-Krezel \& Driever 1994). We were therefore surprised to see extensive EB3-GFP puncta throughout the yolk cell, which could reflect the non-centrosomal origin of some microtubules. During early epiboly, we also observed that the continuity between the upper and lower microtubule network began to diminish, as a region of reduced microtubule density appeared that we call the dim zone (DZ). Due to technical issues, we have so far been unable to simultaneously examine labeled EB3 and microtubules in live embryos which would allow us to understand the timing of these events in greater detail. The DZ was very obvious in dclk2DeltaK-GFP embryos and it was detected in GFP-tuba81 embryos. However, GFP-tuba81 embryos exhibit much lower levels of fluorescence than dclk2DeltaK-GFP embryos, which made the DZ more difficult to characterize.

After DZ formation, the structure of the microtubules in the DZ changed, although the details and molecular bases for these changes remains to be determined. Microtubules in the DZ appeared more diffuse and the overall reduction in fluorescence suggests that some were degraded, which was supported by time-lapse movies in which microtubule fragments entered the DZ and then lost their fluorescence. The DZ became more prominent during mid-epiboly stages as it moved as a wave front towards the vegetal pole as the microtubules vegetal to it shortened. One possibility is that the DZ represents the leading edge of YSL, which replaces the yolk cytoplasmic layer during epiboly. Towards the end of epiboly, the network became disorganized and the DZ was no longer apparent.

Microtubule dynamics are controlled by a number of factors including the tubulin isoforms being expressed (Panda et al. 1994), tubulin post-translational modifications (Janke \& Chloë Bulinski 2011), and the activity of different microtubule associated proteins (MAPs) and motors (Heald \&

403 Nogales 2002; van der Vaart et al. 2009). A possible cause for the appearance of the DZ could be 404 due to cleavage of microtubules by the microtubule severing protein Katanin, which has previously been reported to play a role in YSL epiboly (Bruce \& Sampath 2008). Katanin severing in the upper region of the yolk cell would generate microtubules with free minus ends

407 which would be expected either to be depolymerized or stabilized (Akhmanova \& Hoogenraad 408 2015). Microtubules could be stabilized by the minus end binding protein Camsap2a. Camsaps are a recently identified family of microtubule minus end binding proteins and in other systems, 
410 Camsap2 plays a critical role in stabilizing non-centrosomal microtubules (Akhmanova \&

411 Hoogenraad 2015). Expression of zebrafish camsap2a is first detected in the e-YSL at sphere

412 stage (Xu et al. 2012; Hong et al. 2010) where it may have a similar role. Katanin activity is

413 regulated in a variety of ways and Katanin has been shown to regulate Camsap activity

414 (Akhmanova \& Hoogenraad 2015; Bailey et al. 2015). We postulate that these regulatory

415 mechanisms control the extent of the DZ. During mid-epiboly, we detected a population of

416 detyrosinated microtubules just vegetal to the DZ. Detyrosinated microtubules are relatively

417 long-lived and interestingly, it has been shown that Camsap2 preferentially interacts with

418 detyrosinated microtubules in cultured U2OS cells (Jiang et al. 2014). Detyrosinated

419 microtubules are known to pause their growth due to capping (Infante et al. 2000), which could

420 also contribute to the reduction in EB3-GFP puncta we observed during mid-epiboly. The

421 stabilization of a subset of microtubules, by Camsap or other MAPs, would then enable further

422 stabilization via the accumulation of post-translational modifications (Janke \& Chloë Bulinski

423 2011).

425 We propose that timed expression of MAPs could be involved in the observed changes in 426 microtubule dynamics. At present, the only characterized MAP in the zebrafish yolk cell is 427 Clipla, a zebrafish CLIP-170 homolog (Weng et al. 2013). Work from these authors showed that 428 the steroid pregnenolone is required for normal epiboly and is involved in yolk cell microtubule 429 stabilization (Hsu et al. 2006). They subsequently showed that pregnenolone functions by binding 430 to Clipla which then stimulates microtubule polymerization (Weng et al. 2013). As Clipla is 431 maternally expressed and CLIP-170 has been shown to have a preference for tyrosinated tubulin, 432 it might be involved in the establishment of the network, but this remains to be tested (Ikegami \& 433 Setou 2010; Hsu et al. 2006).

435 Another open question is why the microtubule network is dismantled from the top and bottom.

436 One possibility we are currently pursuing is a potential connection between the DZ and the actin

437 cytoskeleton. The DZ is near the actomyosin cable that constricts to close the blastopore during

438 epiboly (Cheng et al. 2004; Behrndt et al. 2012). In some migratory cells, depolymerizing

439 microtubules can stimulate actin contractility via RhoA, and RhoA can in turn stabilize

440 microtubules (Takesono et al. 2010; Chang et al. 2008; Wojnacki et al. 2014; Palazzo et al.

441 2001). This raises the interesting possibility of cross regulatory interactions between the 
442 microtubule and microfilament networks in the yolk cell that might be important for epiboly. We

443 found that microtubule dynamics change around the same time that the actomyosin band becomes

444 active (Behrndt et al. 2012). In addition, we observed that the DZ moves at a similar rate as the

445 blastoderm, and blastoderm movement is driven by the yolk actomyosin motors. An interaction

446 between these two cytoskeletal networks could help explain why in many examples of defective

447 epiboly, both actin and microtubules are disrupted (Lachnit et al. 2008; Lee 2014).

e-YSN Migration

450 Before e-YSN begin migrating, the DZ forms, a subset of microtubules become detyrosinated 451 and EB3-GFP puncta diminish. Initially, e-YSN move along microtubule branches emanating 452 from microtubule organizing centers producing EB3-GFP puncta and not from regions in 453 between. This suggests that e-YSN nucleate microtubule tracks for other e-YSN to migrate along. 454 Our model is that motor proteins, recruited to the e-YSN by the LINC complex, transport the 455 nuclei through and past the DZ towards the vegetal pole. Thus, a given e-YSN is not linked to 456 one set of microtubules throughout its movement and e-YSN appear to move through and largely 457 beneath the bulk of the microtubules. We also saw that in some case microtubules moved slowly 458 animally, in the opposite direction from migrating nuclei. Taken all together, these data do not 459 support a model in which e-YSN are pulled by microtubule shortening from the vegetal pole.

461 The LINC complex is implicated in nuclear movement in many systems, and in keeping with 462 this, we find that expression of a dominant-negative KASH domain construct impaired 463 directional movement of e-YSN. Although our data do not allow us to define the motor protein 464 involved, we suggest kinesin-1 as a likely candidate. In other systems the plus end directed 465 microtubule motor kinesin-1 has high affinity for detyrosinated microtubules (Ikegami \& Setou 466 2010). Interestingly, recent work using cultured fibroblasts and neurons demonstrated a novel 467 function for kinesin-1 in promoting the formation of detyrosinated microtubules (Yasuda et al.

468 2017). Zebrafish kinesin-1, Kif5Ba, is maternally deposited and expressed throughout 469 development (Campbell \& Marlow 2013). We propose that detyrosination of the network 470 facilitates kinesin-1 interaction with microtubules and that kinesin-1 is recruited to e-YSN via the

471 LINC complex. Additional functional studies, as well as determining the location of the 472 centrosome during e-YSN migration, will help clarify the mechanism of nuclear movement. To 473 date the only KASH domain protein characterized during early zebrafish development is 
474 Lymphoid restricted membrane protein, which is involved in centrosome-nucleus attachment in

475 the zygote (Lindeman \& Pelegri 2012). The LINC complex has been implicated in nuclear

476 migration in the zebrafish retina (Tsujikawa et al. 2007) and several uncharacterized LINC

477 complex genes are present in the genome.

479 Our work supports previous reports on nuclear migration in the C. elegans epidermis, the

480 hypodermis. The hypodermis undergoes epiboly during ventral enclosure and is essential for axis

481 elongation (Williams-Masson et al. 1998; Williams-Masson et al. 1997). Hypodermis

482 morphogenesis involves cell intercalation and microtubule dependent nuclear migration and the

483 hyp7 hypodermal precursor cells are used as a model for nuclear migration (Williams-Masson et

484 al. 1998). The KASH domain protein UNC-83 recruits kinesin-1 to the nuclear envelop to drive

485 nuclear migration along microtubules (Meyerzon et al. 2009). Microtubules are nucleated from

486 nuclei that are initially positioned at the immobile side of the cell such that a parallel array of

487 microtubules forms with the plus ends oriented toward the intercalating side of the cell

488 (Meyerzon et al. 2009). The network is thought to become non-centrosomal since nuclei migrate

489 in association with their centrosomes. However, the centrosome does not lead the migration,

490 consistent with the role of the plus end directed motor kinesin-1 (Meyerzon et al. 2009). The

491 similarities in this system to the zebrafish yolk cell suggest that this mode of nuclear migration

492 may be evolutionarily conserved. Important differences include the size difference between $C$.

493 elegans cells and the zebrafish yolk cell and the presence of microtubule branches in zebrafish.

495 Our findings are summarized and assembled into a time line in Fig. 7. As epiboly starts, the DZ

496 becomes apparent, it then moves towards the vegetal pole during epiboly until the late epiboly

497 when the entire microtubule network becomes disorganized. A subset of detyrosinated

498 microtubules becomes detectable at mid-epiboly as the e-YSN begin to migrate towards the

499 vegetal pole. E-YSN migrate more rapidly than the blastoderm and DZ, and their migration

500 requires the LINC complex.

\section{Conclusions}

503 The YSL is conserved in teleosts and is present in other organisms with meroblastic cleavage

504 including the longnose gar, squid and chicken (Long \& Ballard 2001; Wadeson \& Crawford

505 2003; Arendt \& Nübler-Jung 1999; Nagai et al. 2015). The critical signaling function of the YSL 
might explain why it is necessary for YSN to be distributed over the yolk surface during epiboly. During gastrulation different signals are sent to the dorsal and ventral sides of the blastoderm and gene expression in the YSL is temporally dynamic (Carvalho \& Heisenberg 2010; Sun et al. 2014; Thisse \& Thisse 2004). One proposal is that the YSL provides stabilizing signals that

510 enhance the robustness of gastrulation and help maintain regional expression domains in the

511 blastoderm as widespread cell rearrangements occur (Sun et al. 2014). Later developmental

512 events also depend upon YSL signaling, such as heart morphogenesis (Trinh \& Stainier 2004).

513 The distribution of YSN is also likely to be important for the nutritive function of the yolk cell

514 since, as lecithotrophs, zebrafish rely exclusively on the yolk for the first 5 days of development.

\section{Materials and methods}

\section{Zebrafish strains}

518 Zebrafish (Danio rerio) were maintained under standard conditions. AB,

$519 \operatorname{Tg}($ XlEefla1:dclk2DeltaK-GFP) (gift from Marina Mione) (Sepich et al. 2011), and Tg

520 (XlEefla:eGFP-tuba81) strains were used. Embryos were acquired from natural spawnings,

521 maintained at $25-30^{\circ} \mathrm{C}$ and staged as described (Kimmel et al. 1995). Animals were treated in

522 accordance with the policies of the University of Toronto Animal Care Committee.

\section{C-Syne2a construct}

525 cDNA from 1 day post-fertilization embryos was synthesized using the Protoscript II $1^{\text {st }}$ Strand 526 cDNA Synthesis kit (NEB) following the manufacturer's instructions. The region of zebrafish 527 syn2a encoding the KASH domain was PCR amplified from cDNA using Q5 high fidelity Taq 528 polymerase (NEB) using the forward primer: 5'-CCACCATGCGCTCGTTCTTCTACCGTGT529 3' and reverse primer: 5'-TCATGTTGGAGGAGGGCCGT-3'. The PCR fragment was digested 530 with EcoRI and ligated into EcoRI digested pCS2+ (Rupp et al. 1994). Orientation was 531 confirmed by sequencing (TCAG DNA Sequencing Facility, Hospital for Sick Children).

\section{RNA Synthesis and Microinjection}

534 To generate $h 2 a-g f p, e b 3-g f p$, and c-syne $2 a$ sense RNA, NotI digested plasmids were in vitro 535 transcribed using the SP6 mMESSAGE mMACHINE kit (Ambion). RNAs were purified with 536 the MEGAclear kit (Ambion). Embryos were injected into the yolk of 1-cell stage embryos as 
537 described (Bruce et al. 2003). Doses of injected RNA were: $e b 3-g f p$ (110 pg), h2a-gfp (25 pg),

538 and c-syne $2 a(50 \mathrm{pg})$.

539

\section{Generation of Tg:(XIEefla1:GFP-tuba81) Transgenic Zebrafish}

541 To generate an GFP-tubulin fusion construct, primers were designed to amplify the coding region

542 of zebrafish tubulin, alpha 2 (tuba2). The forward primer was 5'-

543 ATGCGTGAGTGTATCTCCAT-3' and the reverse primer was 5'-

544 CTAATACTCCTCACCTTCCT-3'. RT-PCR was performed on shield stage cDNA and the PCR 545 product was cloned into pGEM-T Easy (Promega). Sequencing revealed that the PCR product 546 corresponded to tubulin alpha 8 like (tuba8l). The tuba8l coding sequence was cloned into the 547 EcoRI site of pCS2+. GFP was PCR amplified from the UAS:eGFP-tuba2 plasmid (Asakawa \& 548 Kawakami 2010) using primers containing BamHI and ClaI restriction sites (forward primer: 5'549 ACGGGATCCGCCACCATGGTGAGCAAGGGCGAGGAGCTG-3' and reverse primer: 5'550 CCGCCGATCGATCTTGTACAGCTCGTCCATGC-3'). Following restriction enzyme digest

551 with BamHI and ClaI, the EGFP coding sequence was cloned in-frame upstream of tuba8l.

553 For transgenesis, GFP-tuba 81 was excised from pCS2+ using BamHI and XhoI restriction sites 554 with the XhoI end blunted and inserted downstream of the elongation factor 1 promoter in the 555 Tol2 vector pT2KXIG $\Delta$ in (Urasaki et al. 2006) using the BamHI and ClaI sites with the ClaI end 556 blunted. Tg:(XlEefla1:GFP-tuba81) transgenic zebrafish were generated using Tol2 transposon557 mediated germline transmission (Kotani et al. 2006). Embryos at the 1-cell stage were injected 558 with transposase RNA and pT2KXIG $\Delta$ in-GFP-tuba81 plasmid and fluorescent embryos were 559 selected at $24 \mathrm{hpf}$ and grown to adulthood. GFP positive embryos from the founder generation 560 were raised to adulthood. The first generation of Tg:(XlEefla1:GFP-tuba81) were genotyped by 561 crossing to wild type fish and collecting embryos at $24 \mathrm{hpf}$. Genomic DNA was prepared from 562 approximately 100 embryos per pair and PCR amplification was performed using Taq

563 polymerase (NEB) (forward primer: 5'-ACGGGATCCGCCAC

564 CATGGTGAGCAAGGGCGAGGAG-3' and reverse primer: 5'-

565 ATGAACTTCAGGGTCAGCTTGC-3'). 
568 The following primary antibodies (1:500 dilution) were used: rabbit anti-tubulin-detyrosinated (AB3201, EMD Millipore), mouse anti- $\gamma$-tubulin clone GTU-88 (T6557, Sigma-Aldrich), and mouse anti- $\alpha$-tubulin DM1A (T6199, Sigma-Aldrich). The following secondary antibodies were used at 1:1000: goat-anti-rabbit Alexa 488 (A-11008, Invitrogen) and goat-anti-mouse Alexa 488 (A-11001, Invitrogen). Microtubule antibody staining was performed as described (Topczewski and Solnica-Krezel, 1999) with the following modifications: embryos were fixed in $3.7 \%$

574 formaldehyde, $0.2 \%$ triton $\mathrm{X}-100$ in microtubule stabilization buffer and fixation time was 1.5

575 hours at room temperature or overnight at $4^{\circ} \mathrm{C}$.

\section{Imaging}

578 Live and fixed embryos were imaged using a Quorum WAVEFX spinning disk, a Zeiss LSM

579510 , or a Leica TCS SP8 confocal microscope. Manually dechorionated live embryos were

580 mounted in 0.4-0.8\% low melt agarose (Invitrogen) and immunostained embryos were mounted

581 in small drops of $80 \%$ glycerol on glass bottom dishes (MatTek).

\section{Fluorescence intensity measurements of the Dim Zone and PIV analysis}

584 Images were acquired with a Leica TCS SP8 laser scanning confocal microscope using a HC PL APO CS2 20x/0.75 IMM (N.A. 0.75) from Tg:(dclk2DeltaK-GFP) and Tg:(X1Eefla1:GFPtuba81) zebrafish. An oval was fit to the embryo and the region outside of the embryo was masked to exclude irrelevant signal and improve the clarity of the fluorescence profiles. Images were divided along the lateral axis in to 8 equally sized bins that ran the length of the animal vegetal pole. The mean animal - vegetal intensity profile of each bin was plotted. A central subdivision was used to create the kymographs. Fluorescence intensity profiles from the kymographs were modeled with a polynomial fit and the local minima was used to define the position of the dim zone.

594 Prior to PIV analysis, stationary background signal was removed by subtracting the mean of all

595 the time points from each time point in the series to better detect changes in intensity. For time lapses of Tg:(dclk2DeltaK-GFP) embryos, analysis was restricted to a region of interest just

597 vegetal to the dim zone and this region of interest was updated for each time point as the dim

598 zone moved vegetally. Images were subdivided into 8x8 pixel interrogation windows with 50\% 
600 rectangular region of interest in the center of the embryo, and images were subdivided into 16x16

601 pixel interrogation windows with 50\% overlap. Each interrogation window was then cross-

602 correlated with its corresponding window from the next time point to determine the direction and

603 magnitude of fluorescence intensity movement. For the analysis of microtubule flows presented

604 in Figure 2, vectors for each time point were summed over 5 consecutive time points to capture

605 persistent movement and minimize noise. For Figure 2 time steps were: embryo 1: 5.2

$606 \mathrm{~min} /$ frame; embryo 2: $6.1 \mathrm{~min} /$ frame, embryo 3: $5.1 \mathrm{~min} /$ frame; embryo $4: 4.9 \mathrm{~min} /$ frame. For the

607 analysis of EB3-GFP comet flows presented in Figure 4, vectors representing movement between

608 individual frames $(7.87 \mathrm{sec} /$ frame $)$ were used. The results presented are the mean displacement

609 overtime in the lateral and A-V directions at each time point over the course of similar stages

610 across embryos.

611

612 YSN tracking and identification

613 e-YSN were identified based on their shape and local absence of fluorescent signal in

614 Tg:(dclk2DeltaK-GFP) and Tg:(X1Eefla1:GFP-tuba81) time-lapse movies and tracked using the

615 ImageJ plugin “Manual Tracking”. e-YSN were identified in H2A-GFP expressed embryos based

616 on shape and position within the Z-stack.

617

618 Acknowledgements

619 AB thanks R. Winklbauer for many helpful discussions and insightful comments on the

620 manuscript. We thank A. Akhmanova, M. Mione and K. Sampath for reagents and Henry Hong

621 for confocal assistance.

622

623

624 No competing interests declared.

\section{Funding}

626 Work in AB's lab is funded by Grant 458019 from the Natural Sciences and Engineering

627 Research Council of Canada.

\section{References}

Akhmanova, A. \& Hoogenraad, C.C., 2015. Microtubule Minus-End-Targeting Proteins Review. Curr Biol, 25(4), pp. R162-R171.

Arendt, D. \& Nübler-Jung, K., 1999. Rearranging gastrulation in the name of yolk: evolution of gastrulation in yolk-rich amniote eggs. Mech dev, 81(1-2), pp.3-22. 
Bailey, M.E., Sackett, D.L. \& Ross, J.L., 2015. Katanin Severing and Binding Microtubules Are Inhibited by Tubulin Carboxy Tails. Biophys J, 109(12), pp.2546-2561.

Bachop, W.E, Schwartz, F.J., 1974. Quantitative nucleic acid histochemistry of the yolk sac

Behrndt, M. et al., 2012. Forces driving epithelial spreading in zebrafish gastrulation. Science, syncytium of oviparous teleosts: Implications for hypotheses of yolk utilization. In: Blaxter J.H.S., editor. The Early Life History of Fishes. Berlin: Springer-Verlag. pp. 345-353.

645 338(6104), pp.257-260.

Bone, C.R. \& Starr, D.A., 2016. Nuclear migration events throughout development. J Cell Sci,

Bruce, A.E.E., 2016. Zebrafish epiboly: Spreading thin over the yolk. Dev Dyn, 245(3), pp.244129(10), pp.1951-1961.

Bruce, A.E.E. \& Sampath, K., 2008. Morphing Morphogenesis. Zebrafish, 5(3), pp.197-200.

Bruce, A.E.E. et al., 2003. The maternally expressed zebrafish T-box gene eomesodermin regulates organizer formation. Development, 130(22), pp.5503-5517.

Bulinski, J.C. \& Gundersen, G.G., 1991. Stabilization of post-translational modification of

Campbell, P.D. \& Marlow, F.L., 2013. Temporal and tissue specific gene expression patterns of the zebrafish kinesin-1 heavy chain family, kif5s, during development. Gene expression patterns, 13(7), pp.271-279.

Carvalho, L. \& Heisenberg, C.-P., 2010. The yolk syncytial layer in early zebrafish development. Trends Cell Biol, 20(10), pp.586-592.

Carvalho, L. et al., 2009. Control of convergent yolk syncytial layer nuclear movement in zebrafish. Development, 136(8), pp.1305-1315.

661 Chang, W., Worman, H.J. \& Gundersen, G.G., 2015. Accessorizing and anchoring the LINC 662 complex for multifunctionality. J Cell Biol, 208(1), pp.11-22.

663 Chang, Y.-C. et al., 2008. GEF-H1 couples nocodazole-induced microtubule disassembly to cell contractility via RhoA. Molec Biol Cell, 19(5), pp.2147-2153.

Chen, S.R. \& Kimelman, D., 2000. The role of the yolk syncytial layer in germ layer patterning in zebrafish. Development, 127(21), pp.4681-4689.

667 Cheng, J.C., Miller, A.L. \& Webb, S.E., 2004. Organization and function of microfilaments 668 during late epiboly in zebrafish embryos. Dev Dyn, 231(2), pp.313-323.

669 Cooper, M.S. \& Virta, V.C., 2007. Evolution of gastrulation in the ray-finned (actinopterygian) fishes. J Exp Zool Part B, 308B(5), pp.591-608.

671 D'Amico, L.A. \& Cooper, M.S., 2001. Morphogenetic domains in the yolk syncytial layer of 
axiating zebrafish embryos. Dev Dyn, 222(4), pp.611-624.

Dupin, I. \& Etienne-Manneville, S., 2011. Nuclear positioning: mechanisms and functions. Int Journal Biochem Cell Biol, 43(12), pp.1698-1707.

Fekany, K. et al., 1999. The zebrafish bozozok locus encodes Dharma, a homeodomain protein essential for induction of gastrula organizer and dorsoanterior embryonic structures. Development, 126(7), pp.1427-1438.

Fekany-Lee, K. et al., 2000. The homeobox gene bozozok promotes anterior neuroectoderm formation in zebrafish through negative regulation of BMP2/4 and Wnt pathways. Development, 127(11), pp.2333-2345.

Feldman, B. et al., 1998. Zebrafish organizer development and germ-layer formation require nodal-related signals. Nature, 395(6698), pp.181-185.

Grady, R.M. et al., 2005. Syne proteins anchor muscle nuclei at the neuromuscular junction. Proc Ntl Acad Sci, 102(12), pp.4359-4364.

Gritsman, K., Talbot, W.S. \& Schier, A.F., 2000. Nodal signaling patterns the organizer. Development, 127(5), pp.921-932.

Gundersen, G.G. \& Worman, H.J., 2013. Nuclear positioning. Cell, 152(6), pp.1376-1389.

Ho, C.Y. et al., 1999. A role for the extraembryonic yolk syncytial layer in patterning the zebrafish embryo suggested by properties of the hex gene. Curr Biol, 9(19), pp.1131-1134.

Hong, S.-K. et al., 2010. Pre-gastrula expression of zebrafish extraembryonic genes. BMC Dev Biol, 10(1), p.42.

Hsu, H.-J. et al., 2006. Pregnenolone stabilizes microtubules and promotes zebrafish embryonic cell movement. Nature, 439(7075), pp.480-483.

Ikegami, K. \& Setou, M., 2010. Unique post-translational modifications in specialized microtubule architecture. Cell Struc Func, 35(1), pp.15-22.

Infante, A.S. et al., 2000. Detyrosinated (Glu) microtubules are stabilized by an ATP-sensitive plus -end cap. J Cell Sci, 113 ( Pt 22), pp.3907-3919.

Ishihara, K. et al., 2014. Microtubule nucleation remote from centrosomes may explain how asters span large cells. Proc Ntl Acad Sci USA, 111(50), pp.17715-17722.

Janke, C. \& Chloë Bulinski, J., 2011. Post-translational regulation of the microtubule cytoskeleton: mechanisms and functions. Nat Rev Molec Cell Biol, 12(12), pp.773-786.

704 Jiang, K. et al., 2014. Microtubule minus-end stabilization by polymerization-driven CAMSAP 
706

707

708

709

710

711

712

713

714

715

716

717

718

719

720

721

722

723

724

725

726

727

728

729

730

731

732

733

734

735

736

737

738

739

Kane, D.A., Warga, R.M. \& Kimmel, C.B., 1992. Mitotic domains in the early embryo of the zebrafish. Nature, 360(6406), pp.735-737.

Kimmel, C.B. \& Law, R.D., 1985. Cell lineage of zebrafish blastomeres. II. Formation of the yolk syncytial layer. Dev Biol, 108(1), pp.86-93.

Kimmel, C.B. et al., 1995. Stages of embryonic development of the zebrafish. Dev Dyn, 203(3), pp.253-310.

Koos, D.S. \& Ho, R.K., 1998. The nieuwkoid gene characterizes and mediates a Nieuwkoopcenter-like activity in the zebrafish. Curr Biol, 8(22), pp.1199-1206.

Kotani, T. et al., 2006. Transposon-mediated gene trapping in zebrafish. Methods, 39(3), pp.199206.

Kreis, T.E., 1987. Microtubules containing detyrosinated tubulin are less dynamic. EMBO J, 6(9), pp.2597-2606.

Lachnit, M., Kur, E. \& Driever, W., 2008. Alterations of the cytoskeleton in all three embryonic lineages contribute to the epiboly defect of Pouff1/Oct4 deficient MZspg zebrafish embryos. Dev Biol, 315(1), pp.1-17.

Lee, S.-J., 2014. Dynamic regulation of the microtubule and actin cytoskeleton in zebrafish epiboly. Biochem Biophys Res Comm, 452(1), pp.1-7.

Lepage, S.E. \& Bruce, A.E.E., 2010. Zebrafish epiboly: mechanics and mechanisms. Int J Dev Biol, 54(8-9), pp.1213-1228.

Lindeman, R.E. \& Pelegri, F., 2012. Localized products of futile cycle/lrmp promote centrosomenucleus attachment in the zebrafish zygote. Curr Biol, 22(10), pp.843-851.

Long, W.L. \& Ballard, W.W., 2001. Normal embryonic stages of the Longnose Gar, Lepisosteus osseus. BMC Dev Biol, 1(1), p.6.

Lüders, J. \& Stearns, T., 2007. Microtubule-organizing centres: a re-evaluation. Nat Rev Molec Cell Biol, 8(2), pp.161-167.

Meyerzon, M. et al., 2009. UNC-83 is a nuclear-specific cargo adaptor for kinesin-1-mediated nuclear migration. Development, 136(16), pp.2725-2733.

Mizuno, T. et al., 1999. Removal of vegetal yolk causes dorsal deficencies and impairs dorsalinducing ability of the yolk cell in zebrafish. Mech Dev, 81(1-2), pp.51-63.

Nagai, H. et al., 2015. Cellular analysis of cleavage-stage chick embryos reveals hidden conservation in vertebrate early development. Development, 142(7), pp.1279-1286.

Ober, E.A. \& Schulte-Merker, S., 1999. Signals from the yolk cell induce mesoderm, neuroectoderm, the trunk organizer, and the notochord in zebrafish. Dev Biol, 215(2), pp.167-181. 
Palazzo, A.F. et al., 2001. mDia mediates Rho-regulated formation and orientation of stable microtubules. Nat Cell Biol, 3(8), pp.723-729.

Panda, D. et al., 1994. Microtubule dynamics in vitro are regulated by the tubulin isotype composition. Proc Ntl Acad Sci, 91(24), pp.11358-11362.

Petry, S. \& Vale, R.D., 2015. Microtubule nucleation at the centrosome and beyond. Nat Cell Biol, 17(9), pp.1089-1093.

Petry, S. et al., 2013. Branching microtubule nucleation in Xenopus egg extracts mediated by augmin and TPX2. Cell, 152(4), pp.768-777.

Rodaway, A. et al., 1999. Induction of the mesendoderm in the zebrafish germ ring by yolk cellderived TGF-beta family signals and discrimination of mesoderm and endoderm by FGF. Development, 126(14), pp.3067-3078.

Rupp, R.A., Snider, L. \& Weintraub, H., 1994. Xenopus embryos regulate the nuclear localization of XMyoD. Genes \& Development, 8(11), pp.1311-1323.

Sepich, D.S. et al., 2011. Wnt/PCP signaling controls intracellular position of MTOCs during gastrulation convergence and extension movements. Development, 138(3), pp.543-552.

Sirotkin, H.I. et al., 2000. Bozozok and squint act in parallel to specify dorsal mesoderm and anterior neuroectoderm in zebrafish. Development, 127(12), pp.2583-2592.

Solecki, D.J. et al., 2004. Par6alpha signaling controls glial-guided neuronal migration. Nat Neurosci, 7(11), pp.1195-1203.

Solnica-Krezel, L. \& Driever, W., 1994. Microtubule arrays of the zebrafish yolk cell:

Song, Y. \& Brady, S.T., 2014. Post-translational modifications of tubulin: pathways to functional organization and function during epiboly. Development, 120(9), pp.2443-2455. Mediated by SUN-KASH Nuclear-Envelope Bridges. Ann Rev Cell Dev Biol, 26(1), pp.421444.

Stepanova, T. et al., 2003. Visualization of microtubule growth in cultured neurons via the use of EB3-GFP (end-binding protein 3-green fluorescent protein). J Neurosci, 23(7), pp.26552664.

Strähle, U. \& Jesuthasan, S., 1993. Ultraviolet irradiation impairs epiboly in zebrafish embryos: evidence for a microtubule-dependent mechanism of epiboly. Development, 119(3), pp.909919. 
in T cells. PLoS ONE, 5(1), p.e8774.

776

777

778

779

780

781

782

783

784

785

786

787

788

789

790

791

792

793

794

795

796

797

798

799

800

801

802

803

804

805

806

807

808

809

810

811

Takesono, A. et al., 2012. Solute carrier family 3 member 2 (Slc3a2) controls yolk syncytial layer (YSL) formation by regulating microtubule networks in the zebrafish embryo. Proc Ntl Acad Sci USA, 109(9), pp.3371-3376.

Thisse, B., Thisse, C., 2004. Fast Release Clones: A High Throughput Expression Analysis. ZFIN Direct Data Submission (http://zfin.org).

Thomas, R.J., 1968. Yolk Distribution and Utilization During Early Development of a Teleost Embryo (Brachydanio Rerio). J Emb Exp Morph, 19(2), pp.203-\&.

Topczewski, J.J. and Solnica-Krezel, L.,1999. Cytoskeletal dynamics of the zebrafish embryo. Methods Cell Biol, 59 p.205 - 226.

Trinh, L.A. \& Stainier, D.Y.R., 2004. Fibronectin regulates epithelial organization during myocardial migration in zebrafish. Dev Cell, 6(3), pp.371-382.

Trinkaus, J.P., 1963. The cellular basis of Fundulus epiboly. Adhesivity of blastula and gastrula cells in culture. Dev Biol, 6, pp.513-532.

Trinkaus, J.P., 1993. The yolk syncytial layer of Fundulus: Its origin and history and its significance for early embryogenesis. J Exp Zool, 265(3), pp.258-284.

Tsujikawa, M. et al., 2007. Mechanism of positioning the cell nucleus in vertebrate photoreceptors. Proc Ntl Acad Sci, 104(37), pp.14819-14824.

Umeshima, H., Hirano, T. \& Kengaku, M., 2007. Microtubule-based nuclear movement occurs independently of centrosome positioning in migrating neurons. Proc Ntl Aca Sci, 104(41), pp.16182-16187.

Urasaki, A., Morvan, G. \& Kawakami, K., 2006. Functional dissection of the Tol2 transposable element identified the minimal cis-sequence and a highly repetitive sequence in the subterminal region essential for transposition. Genetics, 174(2), pp.639-649.

van der Vaart, B., Akhmanova, A. \& Straube, A., 2009. Regulation of microtubule dynamic instability. Biochem Soc Trans, 37(Pt 5), pp.1007-1013.

Virta, V.C. \& Cooper, M.S., 2011. Structural components and morphogenetic mechanics of the zebrafish yolk extension, a developmental module. J Exp Zool Part B: Molec Dev Evo, 316(1), pp.76-92.

Wadeson, P.H. \& Crawford, K., 2003. Formation of the blastoderm and yolk syncytial layer in early squid development. Biol Bull, 205(2), pp.179-180.

Webster, D.R. et al., 1987. Differential turnover of tyrosinated and detyrosinated microtubules. Proc Ntl Acad Sci, 84(24), pp.9040-9044.

Weng, J.-H. et al., 2013. Pregnenolone activates CLIP-170 to promote microtubule growth and cell migration. Nat Chem Biol, 9(10), pp.636-642. 
812 Williams-Masson, E.M. et al., 1998. The cellular mechanism of epithelial rearrangement during 813 morphogenesis of the Caenorhabditis elegans dorsal hypodermis. Dev Biol, 204(1), pp.263814276.

815 Williams-Masson, E.M., Malik, A.N. \& Hardin, J., 1997. An actin-mediated two-step mechanism 816 is required for ventral enclosure of the C. elegans hypodermis. Development, 124(15), $817 \quad$ pp.2889-2901.

818 Wojnacki, J. et al., 2014. Rho GTPases at the crossroad of signaling networks in mammals:

819 impact of Rho-GTPases on microtubule organization and dynamics. Small GTPases, 5(1), 820 p.e28430.

821 Xu, C. et al., 2012. Nanog-like Regulates Endoderm Formation through the Mxtx2-Nodal 822 Pathway. Dev Cell, 22(3), pp.625-638.

823 Yasuda, K. et al., 2017. FUS inclusions disrupt RNA localization by sequestering kinesin-1 and 824 inhibiting microtubule detyrosination. J Cell Biol, 216(4), pp.1015-1034. 
828 Figure 1. Changing yolk cell microtubule dynamics during epiboly.

829 Panels are lateral views with the animal pole to the top. (A) Embryo schematics during epiboly, blastoderm, e-YSN, i-YSN, YSL and yolk cell indicated. (B) Sphere stage embryos. Left: alphatubulin antibody staining, right: $\operatorname{Tg}(\mathrm{XlEef1a1:dclk2DeltaK-GFP)} \mathrm{embryo.} \mathrm{Double} \mathrm{headed} \mathrm{arrow}$ indicates e-YSN nucleating microtubule branches. (C-E"') Live confocal projections of 3 $\mathrm{Tg}$ (XlEefla1:dclk2DeltaK-GFP) embryos from early to late epiboly (left to right). (C') bracket marks the dim zone. (C') Arrowheads indicate e-YSN. (D") Arrowheads indicate gaps between microtubule branches. (E') Arrowhead points to clearing region vegetally. (E') Inset shows magnified view of dim zone, arrowheads indicate microtubule fragments. Scale bar: $100 \mu \mathrm{m}$.

Figure 2. The dim zone moves vegetally and is preceded by animally moving microtubules from early to mid-epiboly.

(A) Dim zone characterization. Masked time lapses showing only the embryo and no background (i) were separated in to 8 equally sized bins (ii). The mean across the lateral axis of each bin was taken and the resulting fluorescence profile was plotted (iii) and the centrally located yellow bin was used for subsequent analyses (arrowhead in "ii"). Minima present between the blastoderm and the vegetal microtubule network of the yolk were used to define the location of the dim zone (iv, top panel) and the position the minima was plotted over time (iv, bottom panel). (B) profile are denoted by horizontal yellow bars. Red bars denote dim zone locations that were false upon inspection and therefore removed from subsequent analyses. Red boxes mark early to midepiboly stages and correspond to the red boxes shown in (C). (C) PIV analyses of A-V directed and laterally directed flow of microtubules from early to mid-epiboly (red boxes). In the first 3 embryos, microtubule flow along the A-V axis (black lines) was directed animally, while lateral microtubule flow (red lines) was approximately zero. In the last embryo, microtubule flow was directed vegetally. Error bars show standard deviation. (D) Mean displacement of the microtubules per minute during early to mid-epiboly along A-V or lateral axes of the embryo. The data points represented in the plots are means for all vectors of a given time point. These sets of points for delta lateral and delta A-V were compared with 2-sided t-test using the matlab function ttest 2 , and $* * *$ indicates $\mathrm{p}<0.0001$. Stages that red boxed regions correspond to: embryo 1: dome- $60 \%$ epiboly; embryo 2: dome- $75 \%$ epiboly; embryo 3: dome- $60 \%$ epiboly; embryo 4 : late sphere- $65 \%$ epiboly.

\section{Figure 3. Detyrosinated microtubules are detected at mid-epiboly.} Sphere stage embryos showing absence of staining in the yolk cell, a small region of the blastoderm is visible at the top. (B) $60 \%$ epiboly stage embryo, detyrosinated tubulin is detected in the blastoderm and in the yolk cell (arrow). Arrowhead indicates e-YSN. Scale bar: $100 \mu \mathrm{m}$.

Figure 4. EB3-GFP reveals extensive microtubule polymerization during early epiboly. (A) Lateral confocal projections of wild-type embryos injected with $e b 3-g f p$ RNA, stages as indicated, animal pole up and slightly left. EB3-GFP fluorescent comets (visible as streaks) extend from the YSL into the YCL from sphere to 50\% epiboly stage. By $60 \%$ epiboly comets were primarily confined to the e-YSL. Arrowhead indicates e-YSN centrosomes. Yellow lines mark boundary between the blastoderm and YSL. Scale bar: $50 \mu \mathrm{m}$. (B) PIV analysis of EB3-

873 GFP fluorescent comet flow. Left panel shows still from single plane time-lapse movie analyzed. 
874

875

876

877

878

879

880

881

882

883

884

885

886

887

888

889

890

891

892

893

894

895

896

897

898

899

900

901

902

903

904

905

906

907
Blastoderm at the top right. Mean flow speed along A-V and lateral axes of the embryo measured as the mean of all vectors in a given time point. Positive flow along the A-V axis represents movement from the animal pole to the vegetal pole. These sets of points for lateral and A-V speed were compared with 2-sided t-test using the matlab function ttest2, and $* * *$ indicates $\mathrm{p}<0.0001$. Right panel shows rose plot of PIV vector angles showing that vectors representing EB3-GFP movement are aligned with the A-V axis and directed vegetally.

\section{Figure 5. E-YSN move along and beneath the microtubule network.}

(A-C) Lateral views with the animal pole to the top. (A) Stills from a confocal time-lapse of a Tg:(XIEefla1:GFP-tuba81) embryo during mid-epiboly. Arrowheads indicate migrating e-YSN forming a chain. Scale bar: $25 \mu \mathrm{m}$. (B) e-YSN migration speeds from confocal-time lapse movies of 6 individual embryos and combined data. Embryos 1-5 were dclk2DeltaK-GFP transgenic embryos and embryo 6 was a GFP-tuba81 transgenic embryo. The time-lapse for embryo 6 was considerably shorter than for the other embryos which may explain the broader speed distribution. (C) Stills from spinning disk confocal time-lapse of Tg:(XIEefla1:GFP-tuba81) embryo injected with $h 2 a-g f p$ RNA. The e-YSN becomes elongated and leading tip of e-YSN becomes pointed during migration (yellow arrows). Scale bar: $10 \mu \mathrm{m}$. (D) Depth coded projection from spinning disk confocal of Tg:(XIEefla1:GFP-tuba81) embryo injected with $h 2 a$ $g f p$ RNA. The e-YSN is positioned largely beneath the microtubule network. Scale bar: $10 \mu \mathrm{m}$.

\section{Figure 6. Overexpression of $c$-syne $2 a$ disrupts e-YSN migration.}

Temporal color coding of selected frames from confocal time lapse movies of $h 2 a-g f p$ injected control and $h 2 a$-gfp plus $c$-syne $2 a$ injected embryos. Typical migrating e-YSN labeled with \#1 in control embryo. In the $c$-syne $2 a$ injected embryo: cell \#1 is overrun by the blastoderm, cell \#2 turns perpendicular to the A-V axis and cell \#3 starts to turn perpendicularly. Similar e-YSN behaviors were observed in $c$-syne $2 a$ injected embryos in 4 independent experiments.

\section{Figure 7. Model and Time-Line}

Lateral views of schematic embryo with YSN and microtubules depicted, animal pole to the top and event timing below. Dashed black lines indicate stages and dashed grey lines indicate beginning and end of a given stage. bThe dim zone is represented by the green shaded area. Exactly when detyrosinated microtubules appear is unclear, as indicated by gray bar and question mark. 
908

\section{Tables}

909

910

Table 1. Mean dim zone movement speeds (towards vegetal pole).

\begin{tabular}{c|c|c|c}
\hline Embryo & Mean speed $(\boldsymbol{\mu m} / \mathbf{m i n})$ & S.E.M. & S.D. \\
\hline $\mathbf{1}$ & 1.046354 & 0.225101 & 1.714318 \\
\hline $\mathbf{2}$ & 0.729579 & 0.405647 & 3.369559 \\
\hline $\mathbf{3}$ & 0.446679 & 0.164669 & 1.296606 \\
\hline $\mathbf{4}$ & 1.325641 & 0.467083 & 2.642221 \\
\hline $\mathbf{5}$ & 0.989935 & 0.392737 & 2.078171 \\
\hline $\mathbf{6}$ & 0.567122 & 0.550748 & 1.907846 \\
\hline Overall & 0.826313 & 0.145526 & 2.351045 \\
\hline
\end{tabular}

911

912 Table 2. Mean e-YSN migration speeds per embryo (towards vegetal pole).

\begin{tabular}{c|c|c|c}
\hline Embryo & Mean speed $(\boldsymbol{\mu m} / \mathbf{m i n})$ & S.E.M. & S.D. \\
\hline $\mathbf{1}$ & 2.463912 & 0.051756 & 0.903886 \\
\hline $\mathbf{2}$ & 1.994247 & 0.069696 & 1.099791 \\
\hline $\mathbf{3}$ & 1.903754 & 0.061245 & 1.021159 \\
\hline $\mathbf{4}$ & 1.779376 & 0.045326 & 0.678379 \\
\hline $\mathbf{5}$ & 1.584370 & 0.029046 & 0.783166 \\
\hline $\mathbf{6}$ & 4.541615 & 0.339505 & 2.352159 \\
\hline Overall & 1.936493 & 0.025346 & 1.084550 \\
\hline
\end{tabular}

913

914

915

916 
917 Supplementary Table 1: Individual mean e-YSN speeds.

\begin{tabular}{|c|c|c|c|c|c|c|}
\hline Embryo & YSN \# & $\begin{array}{l}\text { Mean YSN Speed } \\
\text { (um/min) }\end{array}$ & S.D. & $\begin{array}{l}\text { Mean YSN Speed per } \\
\text { embryo (um/min) }\end{array}$ & S.D. & S.E.M. \\
\hline \multirow{14}{*}{ 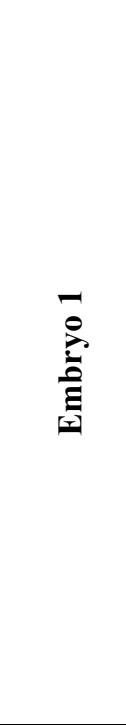 } & 1 & 2.64305 & 0.85958 & \multirow{14}{*}{2.46391} & \multirow{14}{*}{0.90389} & \multirow{14}{*}{0.05176} \\
\hline & 2 & 2.77741 & 0.69480 & & & \\
\hline & 3 & 2.26534 & 0.89144 & & & \\
\hline & 4 & 2.59946 & 0.78143 & & & \\
\hline & 5 & 2.51469 & 0.99693 & & & \\
\hline & 6 & 1.99625 & 0.77162 & & & \\
\hline & 7 & 3.14132 & 0.61522 & & & \\
\hline & 8 & 3.01033 & 0.85603 & & & \\
\hline & 9 & 2.48771 & 0.81211 & & & \\
\hline & 10 & 2.00142 & 0.54466 & & & \\
\hline & 11 & 2.26560 & 0.63658 & & & \\
\hline & 12 & 2.44936 & 0.84536 & & & \\
\hline & 13 & 2.46949 & 1.41007 & & & \\
\hline & 14 & 1.88555 & 0.71601 & & & \\
\hline \multirow{9}{*}{ 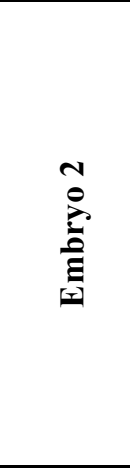 } & 1 & 1.66780 & 0.88297 & \multirow{9}{*}{1.99425} & \multirow{9}{*}{1.09979} & \multirow{9}{*}{0.06970} \\
\hline & 2 & 2.21962 & 0.97129 & & & \\
\hline & 3 & 2.86542 & 0.96104 & & & \\
\hline & 4 & 1.90615 & 1.37631 & & & \\
\hline & 5 & 1.53582 & 0.59896 & & & \\
\hline & 6 & 1.99716 & 1.28856 & & & \\
\hline & 7 & 2.14843 & 1.20684 & & & \\
\hline & 8 & 1.95427 & 0.92599 & & & \\
\hline & 9 & 2.01174 & 0.93745 & & & \\
\hline \multirow{9}{*}{ 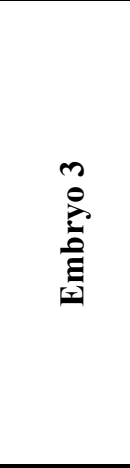 } & 1 & 1.56964 & 0.88809 & \multirow{9}{*}{1.90375} & \multirow{9}{*}{1.02116} & \multirow{9}{*}{0.06125} \\
\hline & 2 & 1.47126 & 0.74640 & & & \\
\hline & 3 & 2.43459 & 1.21290 & & & \\
\hline & 4 & 1.85564 & 1.02315 & & & \\
\hline & 5 & 1.68283 & 1.02704 & & & \\
\hline & 6 & 1.97119 & 0.71362 & & & \\
\hline & 7 & 2.48182 & 0.89896 & & & \\
\hline & 8 & 2.30934 & 1.16967 & & & \\
\hline & 9 & 2.65451 & 1.01697 & & & \\
\hline \multirow{8}{*}{ 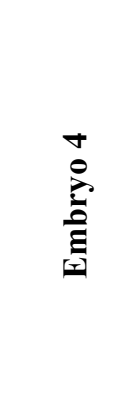 } & 1 & 1.79586 & 0.61101 & \multirow{8}{*}{1.77938} & \multirow{8}{*}{0.67838} & \multirow{8}{*}{0.04533} \\
\hline & 2 & 1.74782 & 0.59338 & & & \\
\hline & 3 & 1.81152 & 0.72883 & & & \\
\hline & 4 & 1.91332 & 0.99313 & & & \\
\hline & 5 & 1.86068 & 0.79398 & & & \\
\hline & 6 & 1.79633 & 0.55116 & & & \\
\hline & 7 & 1.56949 & 0.50300 & & & \\
\hline & 8 & 1.67464 & 0.46723 & & & \\
\hline
\end{tabular}


bioRxiv preprint doi: https://doi.org/10.1101/207795; this version posted October 23, 2017. The copyright holder for this preprint (which was not certified by peer review) is the author/funder. All rights reserved. No reuse allowed without permission.

Supplementary Table 1 continued:

\begin{tabular}{|c|c|c|c|c|c|c|}
\hline Embryo & YSN \# & $\begin{array}{c}\text { Mean YSN Speed } \\
\text { (um/min) }\end{array}$ & S.D. & $\begin{array}{l}\text { Mean YSN Speed per } \\
\text { embryo (um/min) }\end{array}$ & S.D. & S.E.M. \\
\hline \multirow{28}{*}{ 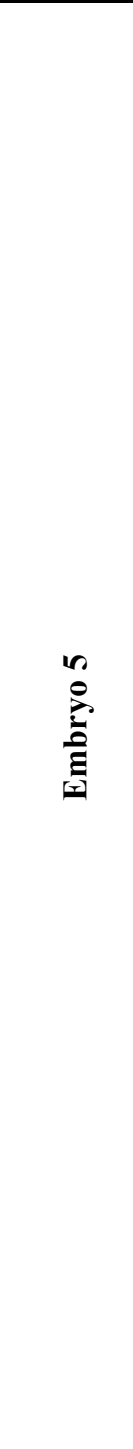 } & 1 & 1.65000 & 0.67455 & \multirow{28}{*}{1.58437} & \multirow{28}{*}{0.78317} & \multirow{28}{*}{0.02905} \\
\hline & 2 & 1.44608 & 0.77222 & & & \\
\hline & 3 & 1.20033 & 0.46432 & & & \\
\hline & 4 & 1.64052 & 0.79410 & & & \\
\hline & 5 & 1.64635 & 0.64747 & & & \\
\hline & 6 & 2.85348 & 1.14968 & & & \\
\hline & 7 & 1.59387 & 0.96490 & & & \\
\hline & 8 & 1.57089 & 0.77610 & & & \\
\hline & 9 & 1.54543 & 0.54685 & & & \\
\hline & 10 & 1.39236 & 0.55407 & & & \\
\hline & 11 & 1.31599 & 0.55331 & & & \\
\hline & 12 & 1.20709 & 0.82376 & & & \\
\hline & 13 & 1.39484 & 0.54749 & & & \\
\hline & 14 & 1.30414 & 0.51379 & & & \\
\hline & 15 & 1.35322 & 0.41103 & & & \\
\hline & 16 & 0.96060 & 0.40852 & & & \\
\hline & 17 & 2.05282 & 0.85517 & & & \\
\hline & 18 & 1.56094 & 0.75835 & & & \\
\hline & 19 & 2.13708 & 0.83124 & & & \\
\hline & 20 & 1.14382 & 0.67141 & & & \\
\hline & 21 & 1.41077 & 0.38285 & & & \\
\hline & 22 & 1.64397 & 0.49593 & & & \\
\hline & 23 & 2.90001 & 0.72388 & & & \\
\hline & 24 & 2.94019 & 0.83398 & & & \\
\hline & 25 & 2.02291 & 0.54649 & & & \\
\hline & 26 & 1.60681 & 0.56840 & & & \\
\hline & 27 & 1.56240 & 0.58647 & & & \\
\hline & 28 & 1.42596 & 0.78403 & & & \\
\hline \multirow{5}{*}{ 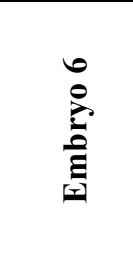 } & 1 & 5.27550 & 3.10518 & \multirow{5}{*}{4.54161} & \multirow{5}{*}{2.35216} & \multirow{5}{*}{0.33950} \\
\hline & 2 & 4.87002 & 2.34267 & & & \\
\hline & 3 & 7.23191 & 1.55758 & & & \\
\hline & 4 & 4.24308 & 1.66159 & & & \\
\hline & 5 & 2.89342 & 1.19383 & & & \\
\hline
\end{tabular}




\section{Supplementary Figure Legends}

\section{Movie 1}

923 Confocal time-lapse movie of Tg(X1Eef1a1:dclk2DeltaK-GFP) embryo from sphere stage to 80\%

924 epiboly. Lateral view with the animal pole to the top.

925

926

\section{Movie 2}

927 Confocal time-lapse movie of embryo expressing EB3-GFP. Lateral view with animal pole to the 928 top.

929

930

\section{Movie 3}

Confocal time-lapse movie of embryo expressing EB3-GFP. e-YSN can be seen emerging from regions where EB3-GFP comets are emanating (arrows). Lateral view, animal pole towards left. Bright region at the top is the blastoderm.

\section{Movie 4}

Spinning disk confocal time-lapse movie of Tg (XlEefla:eGFP-tuba81) embryo with H2A-GFP

940 Confocal time-lapse movie of H2A-GFP expressing control embryo. Lateral view with animal

941 pole towards the upper right.

942

943

944

\section{Movie 6}

Confocal time-lapse movie of H2A-GFP and C-Syne2a expressing embryo. Lateral view with

946

947 
Figure 1

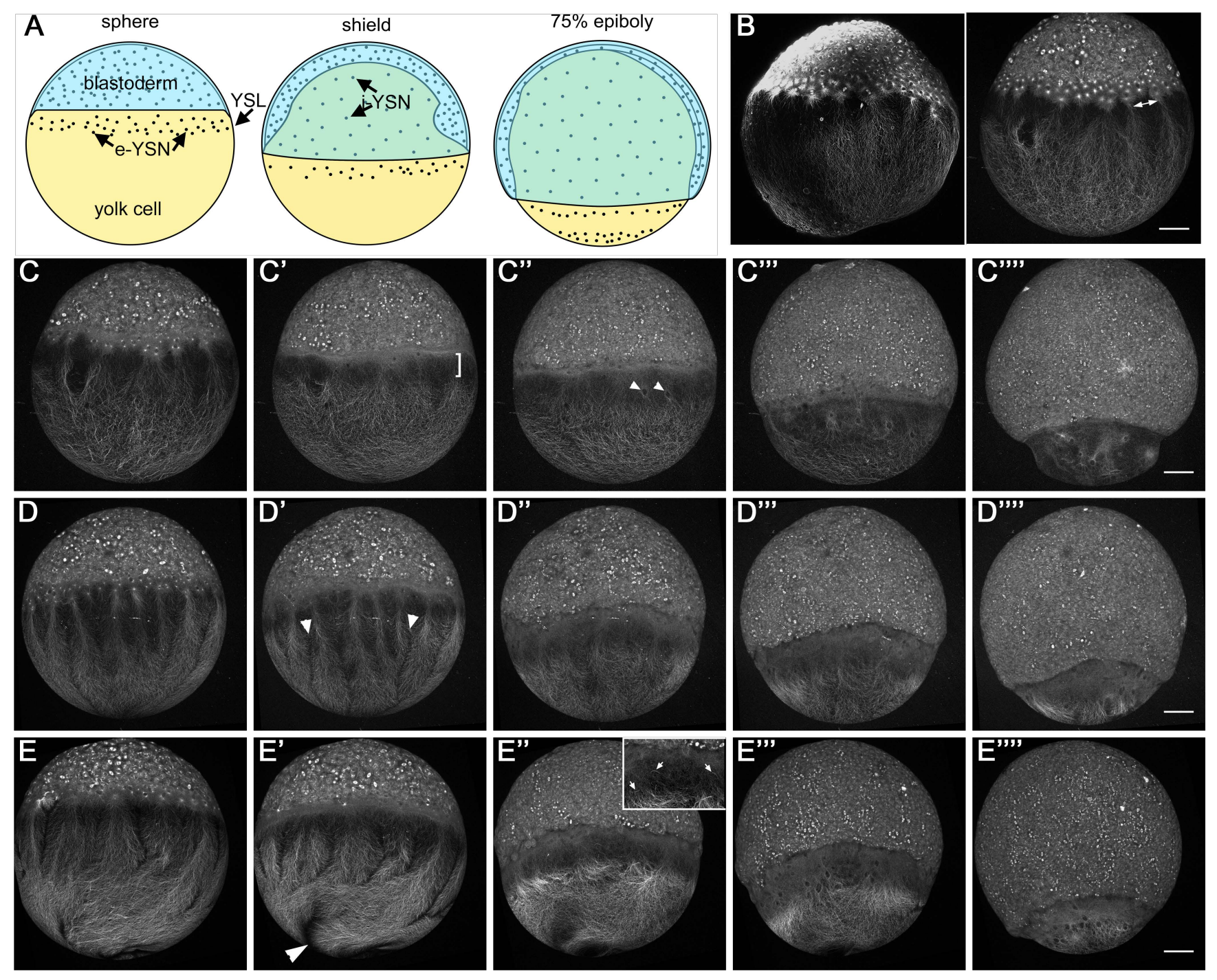


Figure 2

A

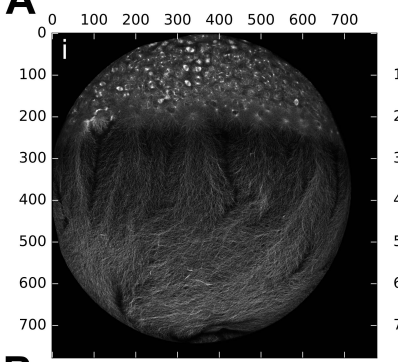

B

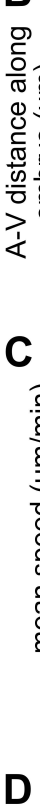

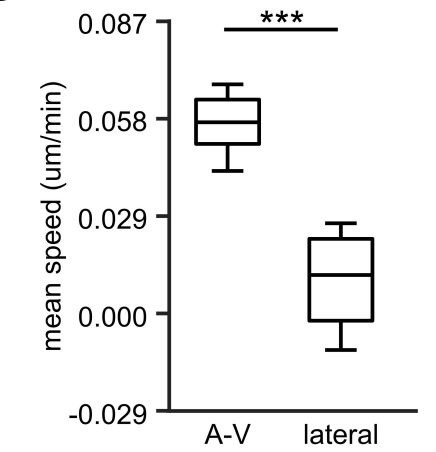

\section{Embryo 1}

time (minutes)

$0^{0} 0^{83} \times 6^{6}+25^{5} 3^{3}$

$$
\text { ) }
$$

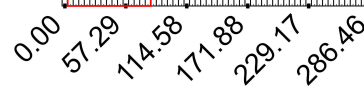

time (minutes)

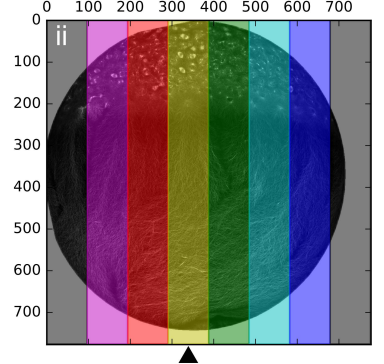

Embryo 2

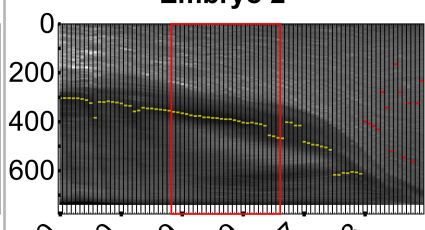

0. time (minutes)
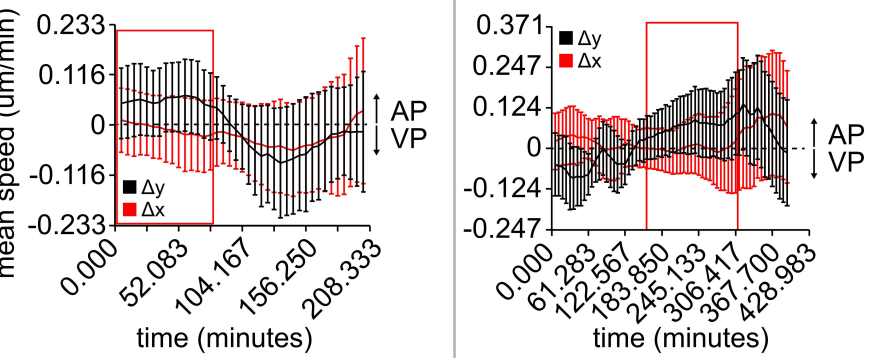

$\Delta y$

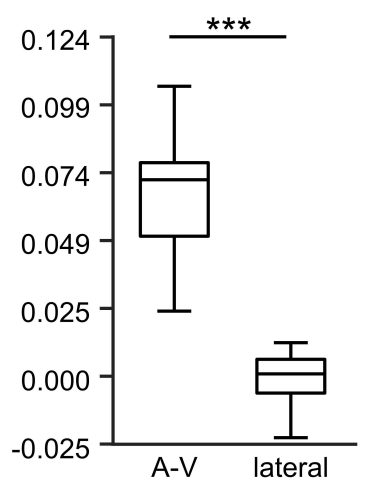

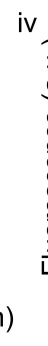

Embryo 3
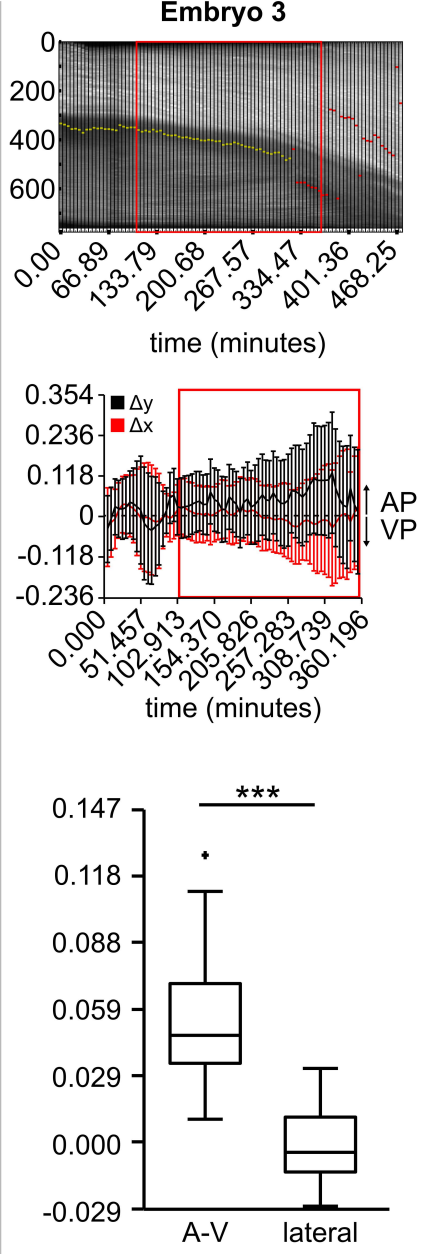

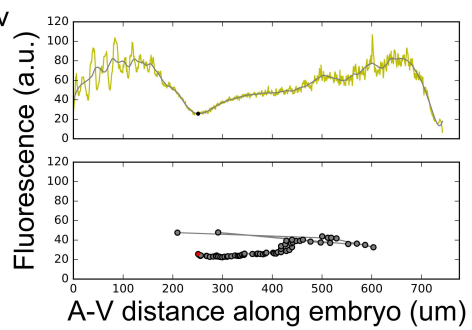

Embryo 4
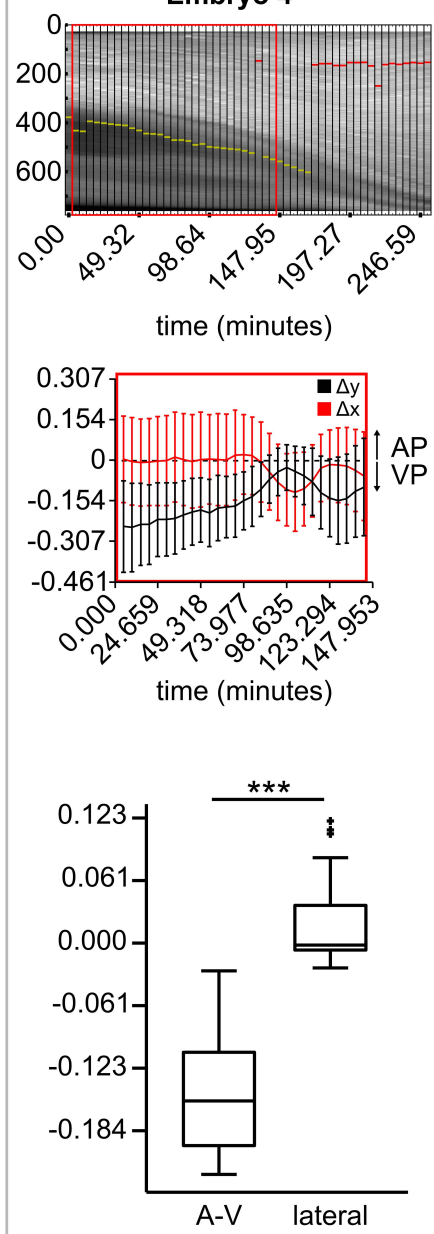


\section{Figure 3}

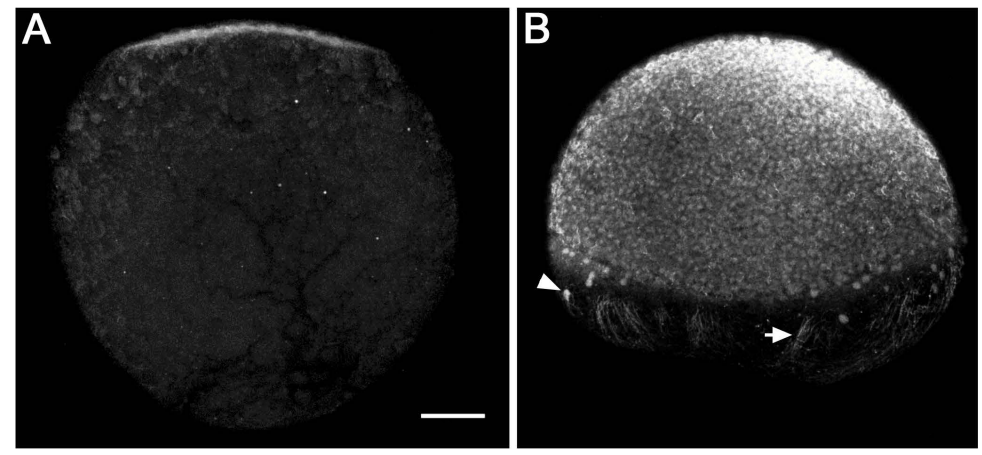




\section{Figure 4}
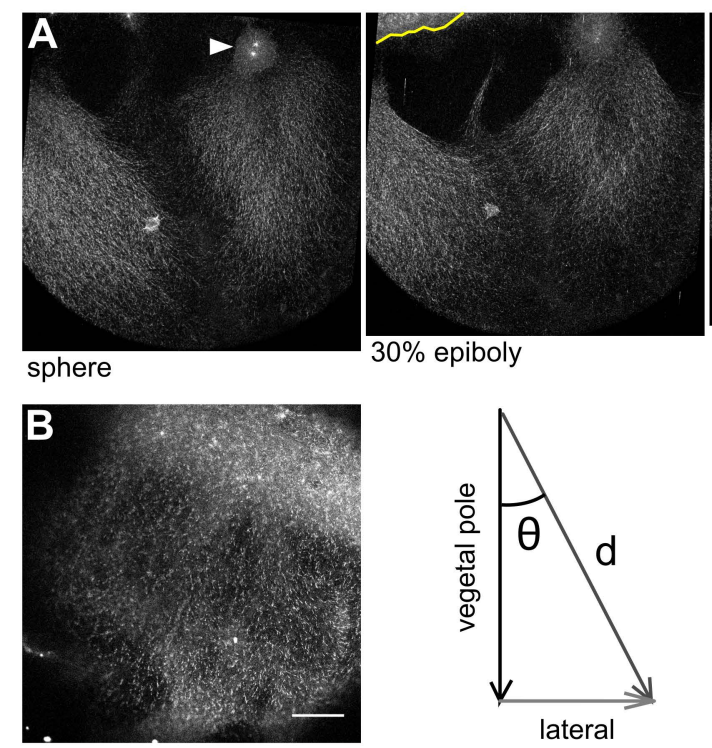
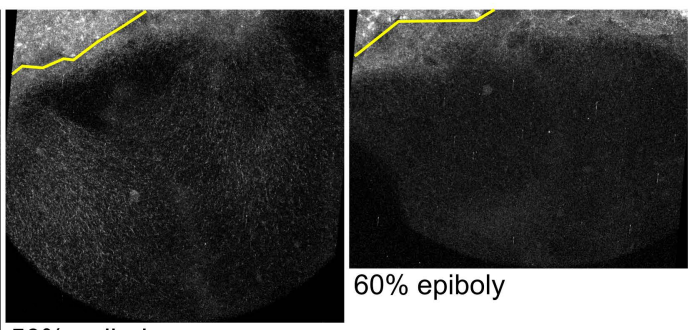

$60 \%$ epiboly

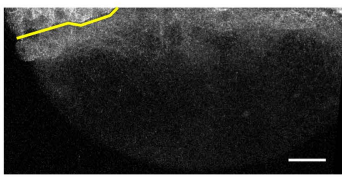

$75 \%$ epiboly
$30 \%$ epiboly

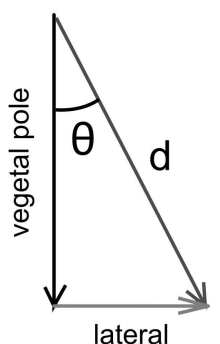

$50 \%$ epiboly

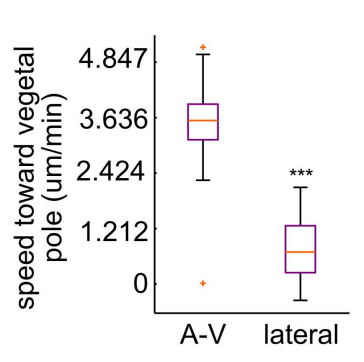

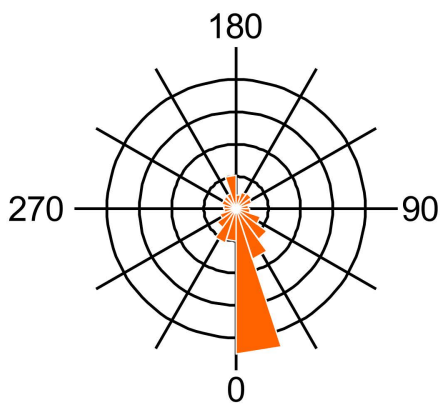


Figure 5
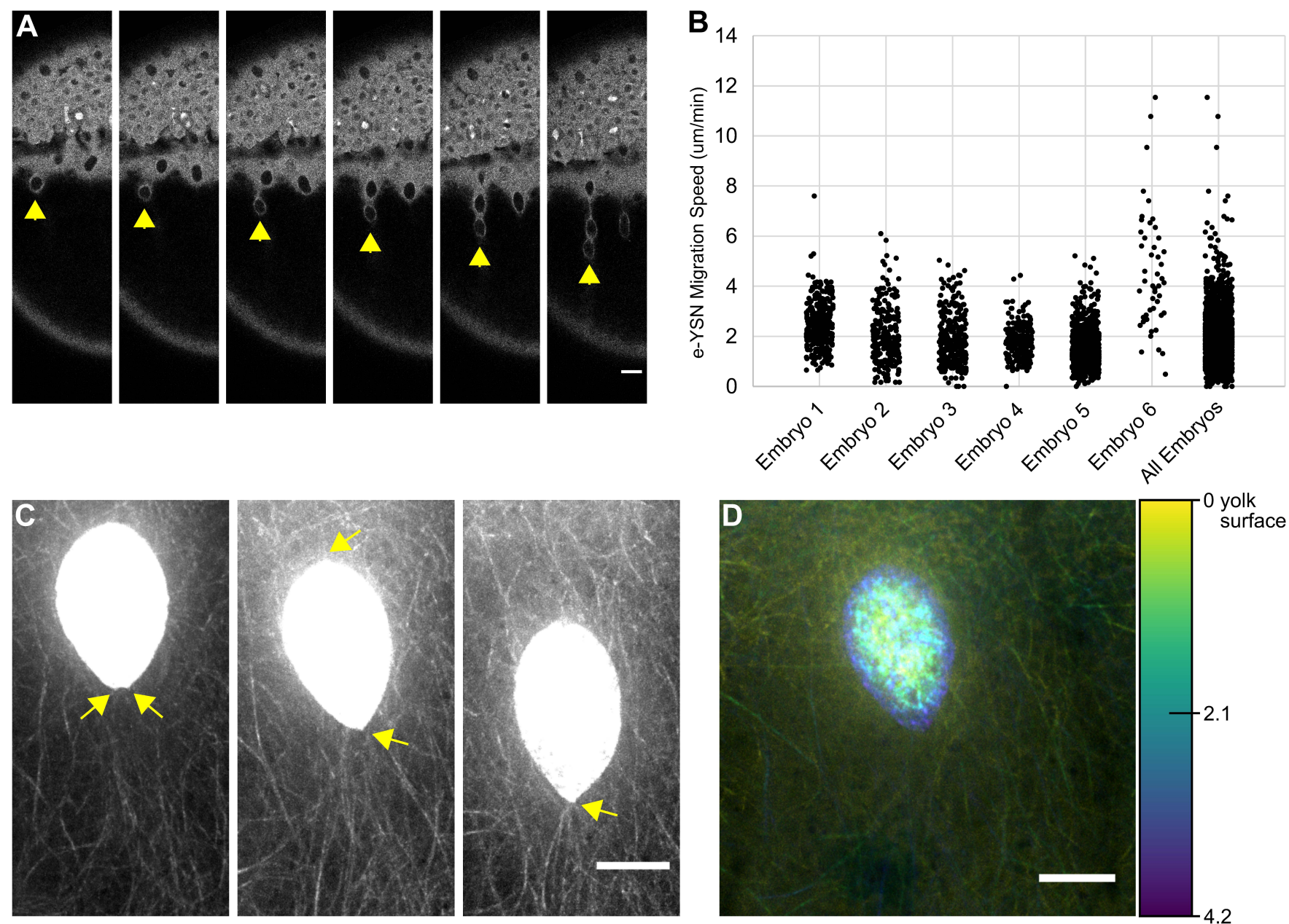
Figure 6

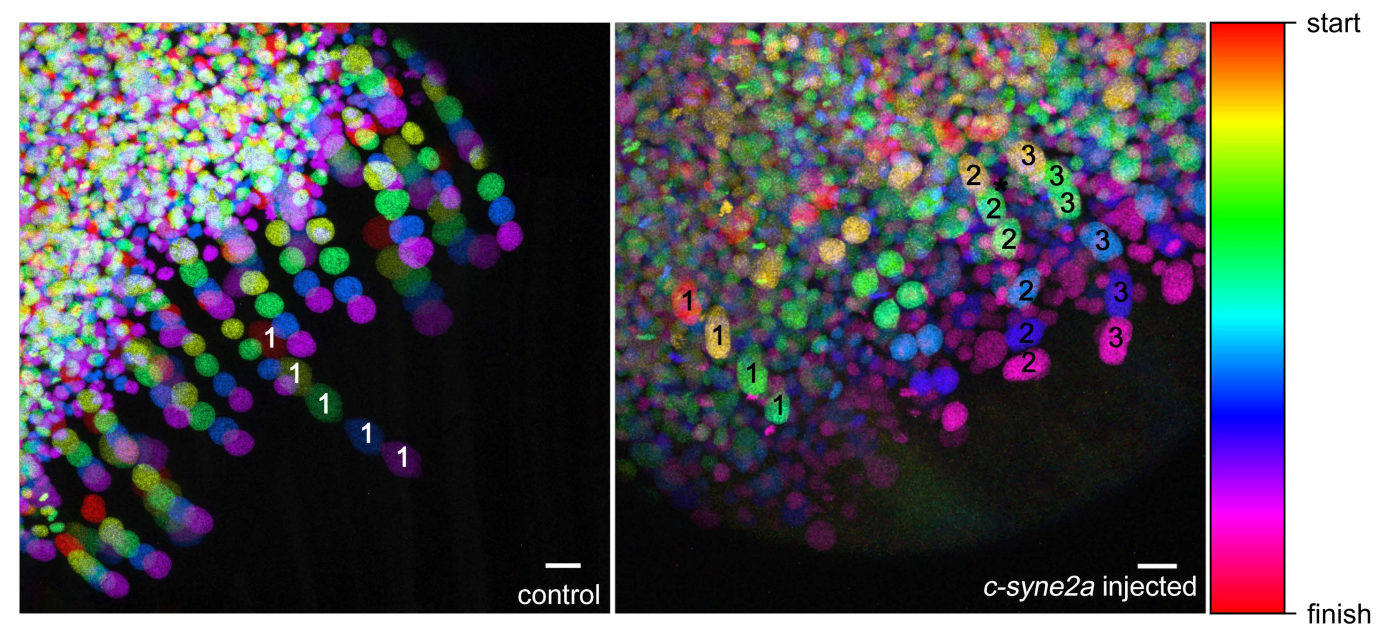




\section{Figure 7}

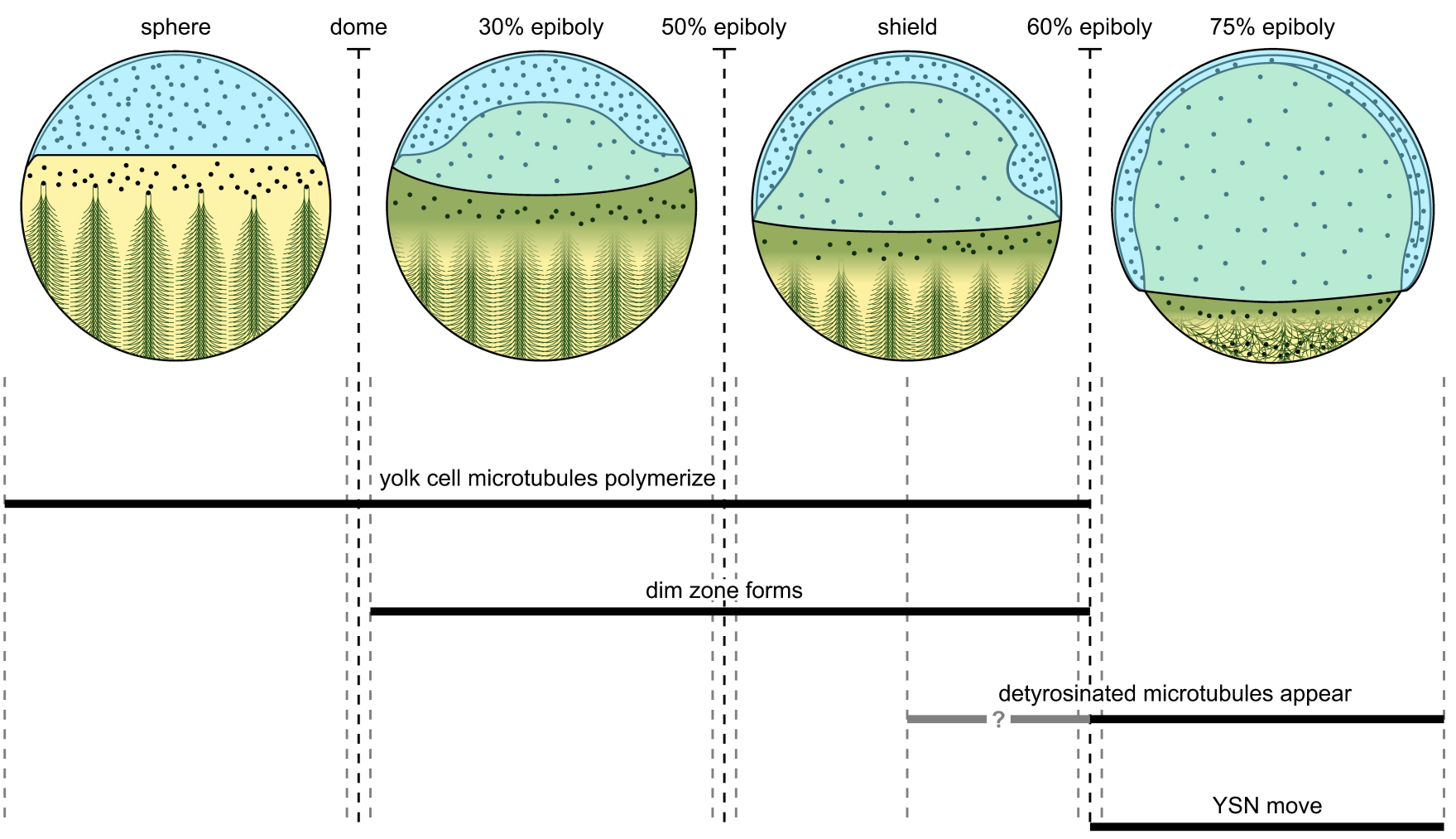

